\title{
Transposition in pigeons: Reassessing Spence (1937) with multiple discrimination training
}

\author{
OLGA F. LAZAREVA and EDWARD A. WASSERMAN \\ University of Iowa, Iowa City, Iowa \\ and \\ MICHAEL E. YOUNG \\ Southern Illinois University, Carbondale, Illinois
}

\begin{abstract}
We studied transposition in pigeons, using multiple-pair discrimination training. Four birds discriminated two pairs of circles: $1+2-$ and $5+6-$ or $1-2+$ and $5-6+$ (digits denote circle diameters and plus and minus signs denote reward and nonreward, respectively). Four other birds discriminated four pairs of circles: $1+2-, 1+3-, 4+6-$, and $5+6-$ or $1-2+, 1-3+, 4-6+$, and $5-6+$. Finally, 4 birds discriminated only one pair of circles: $1+2-, 1-2+, 5+6-$, or $5-6+$. Testing included five new pairs-1/5, 2/3, 2/6, 3/4, and 4/5-that distinguished absolute from relational accounts of transposition. The pigeons' relational responding rose from one- to two- to four-pair training. The similarity of the testing stimuli to one another also affected relational responding: Transposition increased with highly dissimilar stimuli. Neither Spence's (1937) theory nor existing relational accounts could predict the obtained pattern of relational responding.
\end{abstract}

Do animals perceive the relations between or among stimuli? C. Lloyd Morgan (1894) first considered, but later rejected this intriguing possibility, "in no dogmatic spirit, and not in support of any preconceived theory or opinion, but because the evidence now before us is not ... sufficient to justify the hypothesis" (p. 377). Morgan's conservative assessment persists today (Mackintosh, 2000). But it does so in the face of mounting empirical evidence that animals can discriminate the relations between or among stimuli, particularly when collections of several visual stimuli must be judged to be the same as or different from one another (Cook, Cavoto, \& Cavoto, 1995; Cook, Katz, \& Cavoto, 1997; Fagot, Wasserman, \& Young, 2001; Herrnstein, 1990; Thompson \& Oden, 2000; Wasserman, Hugart, \& Kirkpatrick-Steger, 1995; Wright, 1997; Young \& Wasserman, 2001).

\section{Transposition}

Perhaps the most famous chapter in the story of relational discrimination learning in animals comes from the case of transposition, a phenomenon first studied over 80 years ago by the Gestalt psychologist Wolfgang Köh-

The results of this research were partially presented at the 2002 Psychonomic Society annual meeting, at the 2003 Conference on Comparative Cognition, and at the 2004 American Psychological Association meeting. We are most grateful to Al Riley, who encouraged us to conduct these experiments after hearing our reasons for doing so, and to Gregg Oden, who suggested a clever way for us to conduct Experiment 2. Thanks also go to Tina Antes, Kate Freiburger, Andrea Frank, and Brett Gibson for their help in running the experiments and for everyday logistical support. Correspondence concerning this article should be addressed to O. F. Lazareva, E11 Seashore Hall, Department of Psychology, University of Iowa, Iowa City, IA 52242 (e-mail: olga-lazareva@uiowa.edu). ler (1918/1938). Köhler proposed that stimuli are judged not in absolute terms, but relative to one another. Suppose that a pigeon is given a simultaneous visual discrimination task in which the positive discriminative stimulus $(\mathrm{S}+)$ is a $3-\mathrm{cm}$-diameter circle and the negative discriminative stimulus $(\mathrm{S}-$ ) is a $4-\mathrm{cm}$-diameter circle. When the pigeon learns the discrimination and consistently selects the 3 -cm-diameter circle over the $4-\mathrm{cm}$ diameter circle, what precisely has it learned? Has it learned that the 3-cm-diameter circle signals food? Or has it learned that the smaller circle signals food?

If the pigeon learned a relational discrimination, consider how it ought to respond to new pairs of choice stimuli that are presented during a postdiscrimination generalization test. If a $2-\mathrm{cm}$-diameter circle is presented along with a $3-\mathrm{cm}$-diameter circle, the 2-cm-diameter circle will be even smaller than the 3 -cm-diameter $\mathrm{S}+$. Having learned to respond to the smaller stimulus, the pigeon may actually choose the 2-cm-diameter circle (an untrained stimulus) over the 3-cm-diameter circle (the former S+). In his pioneering studies, Köhler (1918/1938) found that chickens and chimpanzees did indeed respond in this relational manner. Köhler called this behavioral phenomenon transposition, by analogy to the fact that the notes of musical melodies do not change their relation to each other when the melodies are moved or transposed to different keys.

Experimental investigations of transposition typically involve (1) training a discrimination between a single pair of stimuli and (2) testing with new pairs of stimuli that lie at different points along the same stimulus dimension (intradimensional generalization testing). Often, transposition has been assessed with stimuli that are pro- 
gressively distant from the training pair: from near tests to far tests of transposition. In any case, a transpositional, or relational, choice is one in which the chosen stimulus bears the same relation to the other stimulus as the $\mathrm{S}+$ bears to the $\mathrm{S}-$; a nontranspositional, or absolute, choice is one in which the chosen stimulus is closer or more similar to the $\mathrm{S}+$.

Experimental studies have usually obtained results that are consistent with transpositional, or relational, responding (reviewed by Reese, 1968; Riley, 1968; Rilling, 1977). Additional evidence from different kinds of discrimination training procedures (e.g., Gonzalez, Gentry, \& Bitterman, 1954; Lawrence \& DeRivera, 1954) is also cited in support of relational responding.

\section{Transposition: Spence's 1937 Gradient Theory}

If finding empirical evidence of transpositional or relational responding were the whole story, there would be no question that animals can perceive the relations between or among stimuli. But there is much more to the story than that. Kenneth W. Spence (1937) devised an ingenious account of discrimination learning that could explain transpositional or relational responding without hypothesizing that animals actually perceive the relations between or among stimuli-a much-celebrated theoretical tour de force. Indeed, both Köhler and Spence were recipients of the American Psychological Association's first Distinguished Scientific Contribution Awards in 1956, in large part for their work in transposition-a fact that reflects the importance of both men's work to our understanding of relational stimulus control.

Spence's (1937) theory of discrimination learning holds that, when a stimulus is associated with reinforcement, a gradient of excitation develops around it. Maximal excitation is produced by the training stimulus, with orderly decreases in excitation occurring as stimuli are increasingly removed from it. In a parallel fashion, when a stimulus is associated with nonreinforcement, a less peaked and broader gradient of inhibition develops around it. If the $\mathrm{S}+$ and the $\mathrm{S}-$ lie along the same stimulus dimension, the excitatory and inhibitory stimulus generalization gradients can algebraically interact to control discriminative responding. To determine the net associative value of any particular stimulus along the dimension, one simply subtracts its inhibitory value (on the inhibitory gradient) from its excitatory value (on the excitatory gradient), yielding a derived postdiscrimination generalization gradient. Actual responding to any pair of stimuli along the stimulus dimension should thus be a function of their net associative values; the higher the net value of one stimulus over the other, the greater the tendency of the organism to respond to it in a choice test.

In fact, the shapes of the excitatory and inhibitory generalization gradients are critical for predicting the occurrence of transposition (Rilling, 1977). When Spence (1937) first proffered his theory, he commented that "the selection of the curves of generalization was more or less arbitrary as little experimental evidence bearing on the problem is available" (p. 434). Many years later, the shapes of the excitatory and inhibitory gradients were carefully documented through extensive empirical research (Ehrenfreund, 1952; Guttman \& Kalish, 1956; Honig, 1962). Excitatory gradients have generally been found to be taller and narrower than inhibitory gradients (reviewed by Honig \& Urcuioli, 1981, and Rilling, 1977; cf. Honig, Boneau, Burstein, \& Pennypacker, 1963; Jenkins, 1965).

Subsequent empirical research has shown that the shape of the derived postdiscrimination stimulus generalization gradient - calculated as the algebraic sum of the experimentally obtained excitatory and inhibitory gradientsgenerally accords with the shape of the empirical postdiscrimination stimulus generalization gradient (Hearst, 1969; Honig, 1962; Marsh, 1972). This result was deemed by many to provide unique and persuasive support for Spence's (1937) theory.

The shape of the empirical postdiscrimination stimulus generalization gradient has been found to have two telltale features (Hanson, 1959; Honig \& Urcuioli, 1981). First, it is asymmetrical; responding on the side of $\mathrm{S}+$ nearer to $\mathrm{S}-$ is much lower than responding on the side of $\mathrm{S}+$ farther from $\mathrm{S}-$ (producing an area shift). Second, the peak of the gradient is not at $\mathrm{S}+$; rather, it is shifted away from S- (producing a peak shift; Purtle, 1973). This second result clearly accords with Spence's (1937) prediction that animals trained with $\mathrm{S}+$ and $\mathrm{S}-$ along the same dimension may respond more, in postdiscrimination stimulus generalization testing, to a novel stimulus than to the $\mathrm{S}+$ with which they were originally trained. Critically, choosing that novel stimulus produces transpositional, or relational, responding, despite the fact that the behavioral mechanism supporting this choice is decidedly nonrelational.

Most theorists agree that Spence's (1937) gradient theory is an elegant and effective account of transposition. Not only does it explain transposition in a nonrelational way, but also it explains the generally weaker transposition effect found in far than in near tests; it does so in terms of the diminishing effect of generalized excitation and inhibition from the $\mathrm{S}+$ and the $\mathrm{S}-$, respectively, to the increasingly distant testing stimuli (see Hebert \& Krantz, 1965; Reese, 1968; Riley, 1968).

Despite the deserved fame of Spence's (1937) theory, it does have shortcomings (reviewed by Riley, 1968). The theory cannot readily account for (1) stronger transposition after simultaneous than after successive discrimination training (e.g., Marsh, 1967; Riley, Ring, \& Thomas, 1960; but see Hebert \& Krantz, 1965; Wills \& Mackintosh, 1999), (2) successful transfer of the intermediate stimulus problem (from $\mathrm{S} 1-, \mathrm{S} 2+, \mathrm{S} 3-$ to $\mathrm{S} 4-$, S5+, S6-; see Riley, 1968, for details), and (3) improved transposition after discrimination training with pairs of multiple S+ and S- stimuli (Johnson \& Zara, 1960; Marsh, 1967).

In our article, we will concentrate on discrimination training that involves multiple $\mathrm{S}+$ and $\mathrm{S}-$ stimuli, because of its unappreciated potential for testing the via- 
bility of Spence's (1937) gradient theory. In addition, the multiple discrimination problem is a simple modification of the same basic task for which Spence's theory was originally devised; it should not require any special extension or modification of Spence's theory to explain the resulting behavioral effects.

\section{Multiple Discrimination Training}

The multiple discrimination problem was developed by Johnson and Zara (1960), who first trained 4-year-old children to simultaneously discriminate black squares of different sizes, $\mathrm{S} 1-$ versus $\mathrm{S} 2+$ and $\mathrm{S} 3-$ versus $\mathrm{S} 4+$, and later tested the children with $\mathrm{S} 4$ versus $\mathrm{S} 5$ (a near pair), S5 versus S6, and S6 versus S7 (two far pairs). A second group of children was trained on a single simultaneous size discrimination, $\mathrm{S} 3-$ versus $\mathrm{S} 4+$, before identical testing. The children trained on the single problem showed a decline in transposition from the near pair to the far pairs, whereas the children trained on the double discrimination showed no such decline. Johnson and Zara suggested (but did not actually prove) that it would be impossible to find excitatory and inhibitory stimulus generalization gradients that could account for transposition to the far pairs following mastery of the double discrimination, but not following mastery of the single discrimination, thus calling into question Spence's (1937) theory. Similar results were reported by Sherman and Strunk (1964; cf. the review by Reese, 1968, pp. 67-80).

Later, Marsh (1967) trained pigeons to discriminate four stimuli of different wavelengths. One group of 12 pigeons was trained with $\mathrm{S} 1+$ versus $\mathrm{S} 2-$ and $\mathrm{S} 3$ + versus $\mathrm{S} 4-$; a second group of 12 pigeons was trained with $\mathrm{S} 1+$ versus $\mathrm{S} 2-$ and $\mathrm{S} 3-$ versus $\mathrm{S} 4+$. Within each group, half of the pigeons were given successive discrimination training, so that the birds could not directly compare two training stimuli. The other half of the pigeons were given simultaneous discrimination training; they were able to directly compare the wavelengths of the training stimuli. In the test, all of the pigeons were given the choice of S2 versus S3. Marsh (1967) found that pigeons trained with the same relation in both pairs of the simultaneous discrimination (S1+ vs. S2 - and $\mathrm{S} 3+$ vs. S4-) selected the previously nonreinforced S2 over the previously reinforced S3 an average of $76 \%$ of the time, whereas all of the other pigeons responded in accord with the absolute reinforcement histories of the discriminative stimuli. Marsh (1967) claimed (but also did not prove) that it is impossible to find excitatory and inhibitory gradients that could account for both discrimination mastery and choice of a previously nonreinforced stimulus (S2-) over the previously reinforced stimulus $(\mathrm{S} 3+)$. This result too calls into question Spence's (1937) theory.

Despite the strong challenges posed by the methods and results of Johnson and Zara (1960) and Marsh (1967), these research projects appear to have had very little effect on evaluations of Spence's (1937) gradient theory (Mackintosh, 1974; Riley, 1968; Rilling, 1977). Consid- ering the unexplored potential of this multiple discrimination approach, we observed that Spence's theory could be exposed to another critical empirical test when the multiple training pairs are separated by untrained testing stimuli.

Suppose, for example, that we concurrently trained two discriminations: $\mathrm{S} 1+$ versus $\mathrm{S} 2-$ and $\mathrm{S} 5+$ versus S6-, where the different numerals represent the diameters of different circles. (The counterbalanced pair of discriminations, S1- vs. S2+ and S5- vs. S6+, can also be trained; we term the first discrimination Smaller+ and the second discrimination Larger +.) After task mastery, we can test all of the experimental animals with S3 versus S4- two stimuli with no prior reinforcement histories. Relational responding would be exhibited by the choice of S3 over S4 after Smaller+ training (and by the choice of S4 over S3 after Larger+ training). Could this pattern of choice behavior eventuate from interacting gradients of excitation and inhibition, as Spence (1937) would suppose?

At first glance, an absolute theory of discrimination learning, such as Spence's (1937), suggests just the $o p$ posite choice of S4 over $\mathrm{S} 3$ following Smaller+ training; after all, S4's nearest trained neighbor is S5 (an S+), whereas S3's nearest trained neighbor is $\mathrm{S} 2$ (an $\mathrm{S}-$ ). Nevertheless, the precise shapes of the gradients of excitation (peaking at S1 and S5) and inhibition (peaking at S2 and S6) might affect the ultimate choice of S3 or S4. Therefore, we proceeded to conduct a series of simulations to confirm the predictions of Spence's theory before embarking on any experimental investigations.

\section{Multiple Discrimination Training: Simulations}

We selected six white circles as our training stimuli, with the diameter of the smallest circle being $0.71 \mathrm{~cm}$ and the increase in diameter from one circle to the next always equaling $0.36 \mathrm{~cm}$. Circle size has been used in several transposition studies with many species (e.g., Mark \& Maxwell, 1969; Pasnak \& Kurtz, 1987), and it has been shown to produce robust stimulus generalization gradients of prototypical shape (Shepard, 1987).

To evaluate the possibility of relational choice eventuating from interacting stimulus generalization gradients, we comprehensively sampled gradients of excitation and inhibition. We used a family of Gaussian distribution functions, because they have been found to be the best approximations of empirical excitatory and inhibitory gradients (Blough, 1969; Rilling, 1977). We plotted individual gradients and their algebraic sum, using Mathematica, Version 4.2 (Wolfram Research, Inc., Champaign, IL, 2002). The selected stimulus generalization gradients shown in Figures 1 and 2 ensured that (1) both excitatory and inhibitory gradients would be symmetrical, (2) the excitatory gradients would be taller and narrower than the inhibitory gradients, (3) the algebraic summation of the excitatory and the inhibitory gradients would produce both positive and negative peak shifts, and (4) the postdiscrimination gradients would support high discrimina- 
tion performance to the $\mathrm{S}+$ and $\mathrm{S}-$ training stimuli. These requirements tightly constrained the family of Gaussian distribution functions. The functions presented here were selected for their adherence to the conditions above, not for their ability to predict relational responding to the novel testing pairs.

Figure 1 demonstrates that our excitatory and inhibitory stimulus generalization gradients satisfied the first two requirements; the symmetrical excitatory gradient was taller and narrower than the symmetrical inhibitory gradient. Figure 2 demonstrates that summation of the excitatory and the inhibitory stimulus generalization gradients produced both positive and negative peak shifts for each of the training pairs (especially for the discriminations involving S5 and S6), as well as producing very effective discrimination of each of the $\mathrm{S}+$ and $\mathrm{S}-$ training stimuli: $\mathrm{S} 1+$ versus $\mathrm{S} 2-, \mathrm{S} 1-$ versus $\mathrm{S} 2+, \mathrm{S} 5+$ versus $\mathrm{S} 6-$, and $\mathrm{S} 5-$ versus $\mathrm{S} 6+$. (Because constant differences in circle diameter are not equivalently discriminable [Larsen \& Bundesen, 1978], we spaced the six circles along a logarithmic scale in Figure 2 and in all the subsequent figures.)

Next, we examined the postdiscrimination predictions that these gradients yielded for the Smaller+ and Larger+ groups trained with Pairs S1 versus S2 and Pairs S5 versus S6. Figures 3A and 4A show the gradients of excitation and inhibition as well as the postdiscrimination gradients of the Smaller+ and Larger+ groups, respectively. We specifically considered the predictions for the following testing pairs: S3 versus S4 (the middle, untrained pair), $\mathrm{S} 1$ versus S5 and S2 versus S6 (pairs of stimuli that were either both reinforced or both nonreinforced in training). and $\mathrm{S} 2$ versus $\mathrm{S} 3$ and $\mathrm{S} 4$ versus $\mathrm{S} 5$ (one stimulus was untrained and the other stimulus was either reinforced or nonreinforced in training).

We first found that, due to the nonlinear spacing of the stimuli along the size dimension, Spence's (1937) theory predicted better discriminative performance on the $\mathrm{S} 1$ versus S2 training pair than on the S5 versus S6 training

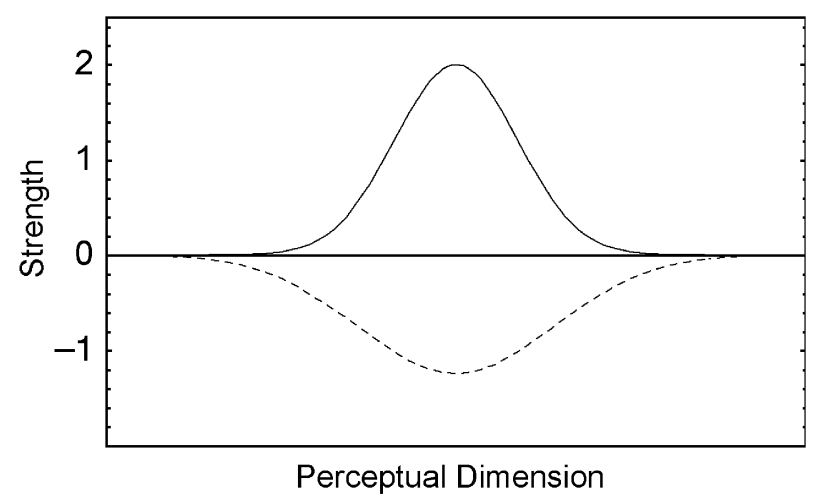

Figure 1. Selected excitatory (smooth line) and inhibitory (dashed line) stimulus generalization gradients for the a priori simulations. pair; it also predicted slightly different results for the different testing pairs in the Smaller+ and Larger+ groups. More important, in both the Smaller+ and the Larger+ groups, the simulations failed to predict relational responding to most of the testing pairs - particularly, S2 versus $\mathrm{S} 3, \mathrm{~S} 3$ versus $\mathrm{S} 4$, and $\mathrm{S} 2$ versus S6. (Table 1 shows the results predicted by Luce's [1959] choice rule from the stimulus generalization gradients depicted in Figures 3 and 4 . In these and most of the other simulations to follow, the bias parameter $A$ of Luce's choice rule was set to 1 [Appendix A, Equation A3].) Critically, for the Smaller+ group, Spence's theory predicts a choice of S4 over S3, whereas for the Larger+ group, Spence's theory predicts a choice of $\mathrm{S} 3$ over $\mathrm{S} 4$ - in each case, the opposite of relational responding.

Of course, gradient shape might play a critical role in these simulated results. We had to be sure that our assessment of Spence's (1937) theory was suitably general. So we next considered three deviations from the first set of idealized excitatory and inhibitory stimulus generalization gradients: (1) the case in which the inhibitory gradient was taller and narrower than the excitatory gradient (Figures 3B and 4B), (2) the case in which both gradients were as tall and narrow as the excitatory gradient in our original simulation (Figures $3 \mathrm{C}$ and $4 \mathrm{C}$ ), and (3) the case in which both gradients were as short and wide as the inhibitory gradient in our original simulation (Figures 3D and 4D). Although the precise predictions from these three follow-up simulations differed slightly from one another, none of these simulations could account for relational responding to all five of the testing pairs: S3 versus S4, S1 versus S5, S2 versus S6, S2 versus S3, and S4 versus S5 (see Table 1). For example, for $\mathrm{S} 3$ versus $\mathrm{S} 4$, all of the simulations predicted the absolute choice of S3 in the Larger+ group and the absolute choice of S4 in the Smaller+ group. Uniformly absolute predictions were also made for $\mathrm{S} 2$ versus $\mathrm{S} 3$ and $\mathrm{S} 2$ versus $\mathrm{S6}$.

Beyond these considerations, it can be shown that, for stimulus generalization gradients based on radially symmetric functions to produce any hint of relational responding to Testing Pairs S3 versus S4, S2 versus S3, and S4 versus $\mathrm{S} 5$, the excitatory and inhibitory gradients must be extremely broad, so that the intermediate testing stimuli can inherit adequate generalized associative strength from the training stimuli. Figure 5 illustrates a case in which the gradients of excitation and inhibition are broad enough to supply approximately equal levels of generalized strength to $\mathrm{S} 3$ and $\mathrm{S} 4$, so that the postdiscrimination gradients predict near-chance responding to members of that pair. Importantly, such broad stimulus generalization gradients fail to predict high levels of discriminative performance to the training stimuli. Calculating predicted responding to the training pairs from the postdiscrimination gradients shown in Figure 5, we obtained chance responding to Training Pair S5 versus S6 and less than $60 \%$ correct responding to Training Pair S1 versus S2. Thus, broadening the stimulus generalization gradients 


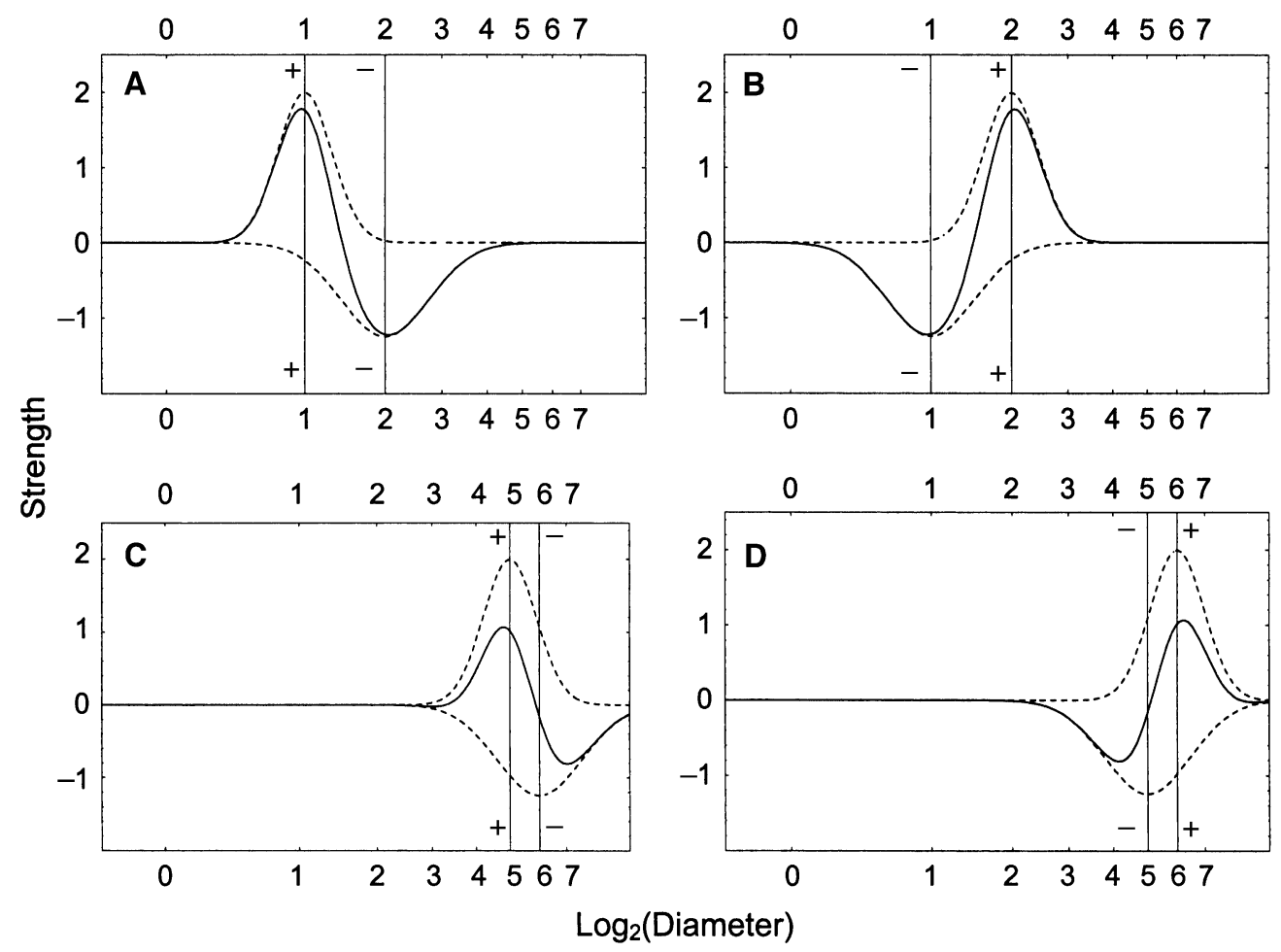

Figure 2. Gradients of excitation and inhibition (dashed lines) and postdiscrimination gradients (smooth lines) for (A) Pair S1 + S2 - , (B) Pair S1 - S2 +, (C) Pair S5 + S6-, and (D) Pair S5- S6 + . All of the postdiscrimination gradients show positive and negative peak shifts, although these effects are larger in $\mathrm{C}$ and $D$ than in $A$ and $B$.

may help to predict relational responding to $\mathrm{S} 3$ versus $\mathrm{S} 4$, but this benefit comes at the cost of failing to predict a high level of discriminative responding to the training stimuli. Moreover, empirical research has shown that both excitatory and inhibitory stimulus generalization gradients become steeper with training (Hearst, 1969; Heinemann \& Rudolph, 1963; Rilling \& Budnik, 1975; Sidman, 1961). Therefore, we would not expect to obtain such broad stimulus generalization gradients after discrimination mastery.

All of these considerations and simulations led us, in Experiment 1, to give pigeons two-pair discrimination training and, following discrimination mastery, to show them several pairs of testing stimuli. According to Spence's (1937) theory, the pigeons should respond in a nonrelational manner to most of the testing pairs. According to a relational-learning account, all of these testing pairs should support relational responding.

\section{EXPERIMENT 1}

\section{Method}

\section{Subjects}

The subjects were 4 feral pigeons (Columba livia) maintained at $85 \%$ of their free-feeding weights by controlled daily feeding. Grit and water were available ad lib in the home cages. The pigeons had served in unrelated studies prior to the present experiment.

\section{Apparatus and Stimuli}

The experiment used four operant conditioning chambers and Macintosh computers detailed by Wasserman et al. (1995). One wall of each chamber contained a large opening, $7 \times 7 \mathrm{~cm}$, with a frame attached to the outside that held a clear touch screen. An aluminum panel in front of the touch screen allowed the pigeons access to circumscribed portions of a video monitor behind the touch screen. A food cup was centered on the rear wall level with the floor. A food dispenser delivered 45-mg food pellets through a vinyl tube into the cup. A houselight on the rear wall provided ambient illumination during the session.

Two $3.5 \times 3.5 \mathrm{~cm}$ areas, or buttons, positioned on the left and right sides of the central display area, were used to display the circular discriminative stimuli; the rest of the central area was black. The distance between the buttons was $1.1 \mathrm{~cm}$; the distance from the top side of the button to the upper border of the aluminum panel was $2 \mathrm{~cm}$. Responses that occurred outside of the buttons were not recorded; only pecks made within the buttons were recorded and could advance the trial.

The discriminative stimuli were six white circles drawn on a black rectangular background. The diameter of smallest circle, $\mathrm{S} 1$, was $0.71 \mathrm{~cm}$, and the diameter of the largest circle, $S 6$, was $2.51 \mathrm{~cm}$; the increase in diameter from one circle to the next was $0.36 \mathrm{~cm}$. The size of the background was $3.5 \times 3.5 \mathrm{~cm}$. The stimuli were made in Canvas Standard Edition, Version 7.0 (Deneba Software, Miami, FL) and were saved as PICT files with 144 dpi resolution.

\section{Design}

The pigeons were trained to discriminate the two end pairs of circles: S1 versus S2 and S5 versus S6. The birds were randomly assigned to the Smaller+ group $(n=2)$ and to the Larger+ group 

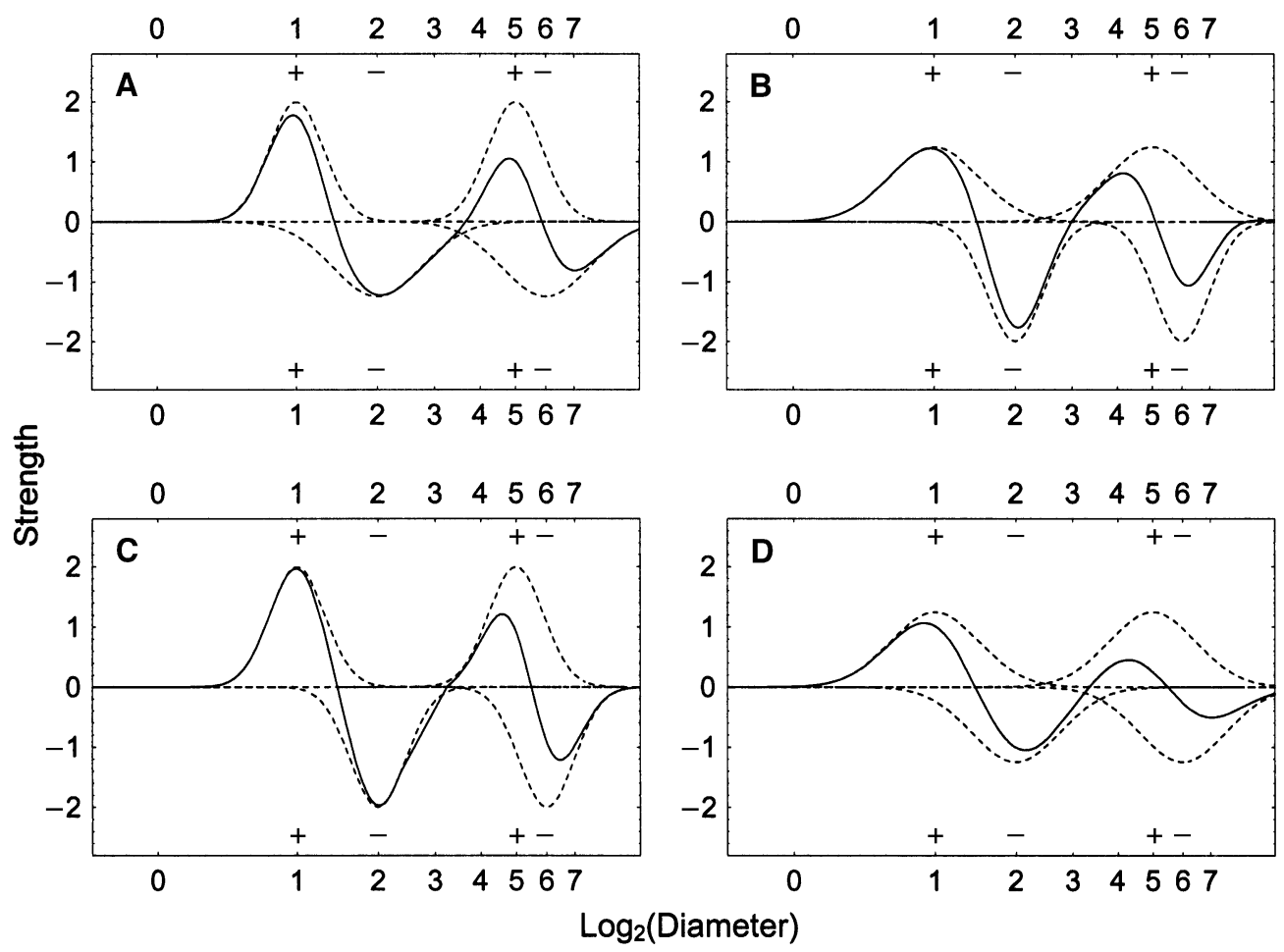

Figure 3. Postdiscrimination gradients (smooth lines) obtained by summation of the excitatory and the inhibitory gradients (dashed lines) for the Smaller+ group trained to discriminate S1 versus S2 and S5 versus S6 (Experiment 1). In A, the excitatory gradient is taller and narrower than the inhibitory gradient; in $B$, the inhibitory gradient is taller and narrower than the excitatory gradient; in $C$, both gradients are as tall and narrow as the excitatory gradient in $\mathrm{A}$; and in $\mathrm{D}$, both gradients are as shallow and short as the inhibitory gradient in A. Note that none of the gradient combinations can account for relational responding to all of the to-be-tested pairs.

$(n=2)$. For the Smaller + group, the smaller of two simultaneously presented circles was always correct; thus, these pigeons were trained to discriminate $\mathrm{S} 1+$ versus $\mathrm{S} 2-$ and $\mathrm{S} 5+$ versus $\mathrm{S} 6-$ (where plus and minus indicate rewarded and nonrewarded stimuli, respectively). The pigeons in the Larger + group were trained to discriminate $\mathrm{S} 1-$ versus $\mathrm{S} 2+$ and $\mathrm{S} 5-$ versus $\mathrm{S} 6+$. This counterbalancing was conducted to control for possible size preferences.

After training, the pigeons were tested with five novel pairs of stimuli. In Test 1 , the middle pair, $\mathrm{S} 3$ versus $\mathrm{S} 4$, was presented. In Test 2, the middle pair, S3 versus $\mathrm{S} 4$, was again presented along with two new pairs: S1 versus S5 and S2 versus S6. Finally, in Test 3 , the three previously tested pairs-S3 versus $\mathrm{S} 4, \mathrm{~S} 1$ versus $\mathrm{S} 5$, and $\mathrm{S} 2$ versus $\mathrm{S} 6$ - were presented along with two new pairs: S2 versus $\mathrm{S} 3$ and $\mathrm{S} 4$ versus $\mathrm{S} 5$.

\section{Procedure}

Pretraining. Following weight reduction, the pigeons began pretraining. Either the left or the right button (a black cross in the middle of a white square area) was illuminated on each trial. The birds were required to make a single peck at the available button to obtain food.

Training. Following pretraining, the birds entered the training phase, in which they were taught to discriminate two pairs of circles, always selecting either the larger or the smaller one. At the beginning of a trial, the pigeons were shown a black cross in the center of the white display screen. Following one peck anywhere on the screen, the two training stimuli appeared for a fixed interval of $5 \mathrm{sec}$; after that time had elapsed, the pigeons were required to make a single peck to one of the stimuli. We gave the pigeons $5 \mathrm{sec}$ to view the two choice stimuli to enhance the opportunity for the birds to attend to both of them. Because the sizes of the choice stimuli differed, a peck within either of the two $3.5 \times 3.5 \mathrm{~cm}$ square areas aligned with the circular stimuli advanced the trial. The stimulus to which the response had been made remained on for $2 \mathrm{sec}$ while the other stimulus was turned off; we arranged this contingency to enhance the birds' attending to the stimulus that they had pecked.

If the choice response was correct, food reinforcement was delivered, and the intertrial interval (ITI) ensued. The ITI varied from bird to bird and ranged randomly from 10 to $25 \mathrm{sec}$. This requirement was adjusted on the basis of the performance of the pigeons. If the bird was consistently pecking but not meeting criterion in a timely fashion, the ITI was increased to make incorrect responses more punishing. If the bird was failing to complete sessions or its accuracy decreased due to our scheduling of longer ITIs, the ITI was decreased.

If the choice response was incorrect, the houselight darkened, and a correction trial was given. On correction trials, the ITI varied randomly from 15 to $30 \mathrm{sec}$ in duration. Correction trials were given until the correct response was made. Only the first report response was scored and used in later data analysis, although correction trials were recorded as well. Occasional incomplete sessions were not used in data analysis; such sessions were most likely at the beginning of training, when errors were prevalent.

During training, each session comprised 200 trials, containing 25 blocks of 8 trials each. Each 8 -trial block included 4 trials of Pair S1 versus S2 and 4 trials of Pair S5 versus S6, with the left-right locations of the stimuli counterbalanced. All the pigeons were required to meet an $85 / 80$ criterion ( $85 \%$ correct overall and $80 \%$ cor- 


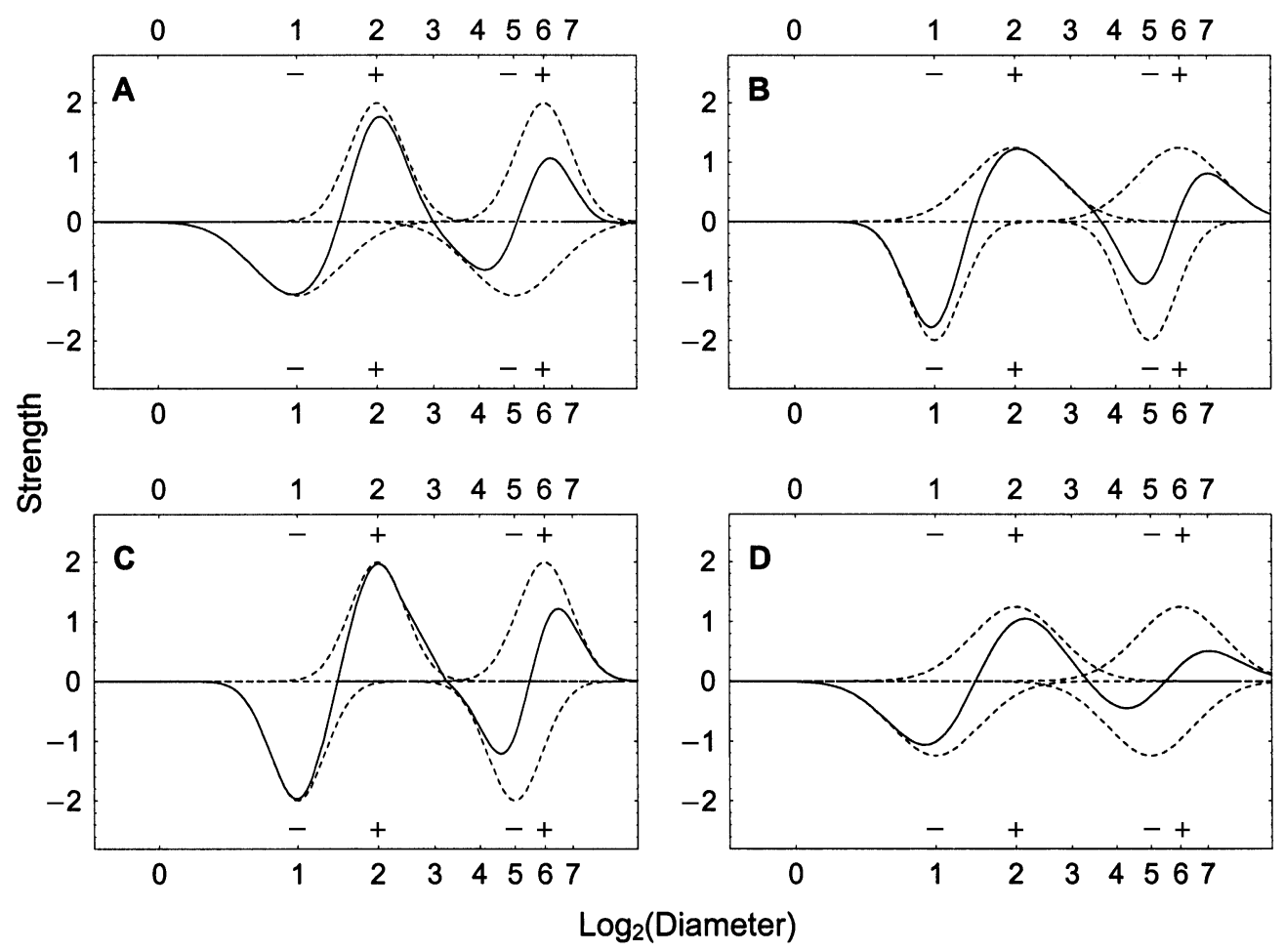

Figure 4. Postdiscrimination gradients (smooth line) obtained by summation of the excitatory and the inhibitory gradients (dashed lines) for the Larger+ group trained to discriminate S1 versus S2 and S5 versus $\mathbf{S 6}$ (Experiment 1). In A, the excitatory gradient is taller and narrower than the inhibitory gradient; in $\mathrm{B}$, the inhibitory gradient is taller and narrower than the excitatory gradient; in $\mathrm{C}$, both gradients are as tall and narrow as the excitatory gradient in $\mathrm{A}$; and, in $\mathrm{D}$, both gradients are as shallow and short as the inhibitory gradient in A. Note that none of the gradient combinations can account for relational responding to all of the to-be-tested pairs.

rect to each of the four possible combinations of stimuli) except Bird 23R; because this pigeon did not reach the $85 / 80$ criterion, its criterion was lowered to $80 / 75$ (80\% correct overall and $75 \%$ correct to each stimulus combination). The selected criterion had to be maintained during testing; if a bird's discrimination performance

Table 1

Percentages of Relational Responding to the Training and Testing Pairs After Two-Pair Discrimination

Training (Experiment 1) Obtained From Simulated

Postdiscrimination Gradients, Using Luce's Choice Rule (Appendix A, Equation A3)

Simulation 1 vs. 2 1 vs. 5 2 vs. $3 \quad 3$ vs. 4 4v. $5 \quad 2$ vs. $6 \quad 5$ vs. 6

\begin{tabular}{llllllll}
\multicolumn{7}{c}{ Smaller+ (Figure 3) } \\
A & 95.17 & 68.05 & $\mathbf{3 4 . 1 4}$ & $\mathbf{2 7 . 8 1}$ & $\mathbf{3 5 . 0 9}$ & $\mathbf{2 5 . 9 3}$ & 76.41 \\
B & 95.12 & 74.07 & $\mathbf{1 4 . 6 7}$ & $\mathbf{3 1 . 4 2}$ & 65.03 & $\mathbf{3 2 . 3 7}$ & 76.57 \\
C & 98.08 & 74.07 & $\mathbf{1 4 . 6 7}$ & $\mathbf{2 7 . 4 4}$ & $\mathbf{4 6 . 2 2}$ & $\mathbf{2 5 . 9 3}$ & 86.23 \\
D & 88.26 & 68.05 & $\mathbf{3 4 . 1 4}$ & $\mathbf{3 1 . 8 3}$ & 53.92 & $\mathbf{3 2 . 3 7}$ & 62.83 \\
\multicolumn{7}{c}{ Larger+ (Figure 4) } \\
A & 95.12 & 74.07 & $\mathbf{1 4 . 6 7}$ & $\mathbf{3 1 . 4 2}$ & 65.03 & $\mathbf{3 2 . 3 7}$ & 76.57 \\
B & 95.17 & 68.05 & $\mathbf{3 4 . 1 4}$ & $\mathbf{2 7 . 8 1}$ & $\mathbf{3 5 . 0 9}$ & $\mathbf{2 5 . 9 3}$ & 76.41 \\
C & 98.08 & 74.07 & $\mathbf{1 4 . 6 7}$ & $\mathbf{2 7 . 4 4}$ & $\mathbf{4 6 . 2 2}$ & $\mathbf{2 5 . 9 3}$ & 86.23 \\
D & 88.26 & 68.05 & $\mathbf{3 4 . 1 4}$ & $\mathbf{3 1 . 8 3}$ & 53.92 & $\mathbf{3 2 . 3 7}$ & 62.83 \\
\hline
\end{tabular}

Note-Letters in the left column correspond to the simulations shown in Figures 3 and 4. Bold font denotes nonrelational choice, and italicized font denotes the training pairs. fell below criterion, it was returned to training until it once again reached criterion.

Test 1. In this test, we presented the middle pair, composed of S3 and S4, which the birds had never seen before. During testing sessions, the birds received 184 daily trials consisting of nine blocks. The first block contained 16 warm-up training trials, with each stimulus combination shown four times; these warm-up trials were not used in statistical analyses. The next eight blocks contained a mixture of training and testing trials. Each block included 20 training trials and 1 testing trial, so that during the entire testing session, the pigeons received four presentations of S3-S4 and four presentations of S4-S3. Testing lasted for 10 days, in order for the pigeons to receive 40 exposures to each left-right combination: S3-S4 and S4-S3.

For the training stimuli, the birds received food only after a correct response; incorrect responses led to one or more correction trials (differential reinforcement). For the testing stimuli, the birds received food after all choice responses (nondifferential reinforcement). We reinforced choices to any of the testing stimuli to guarantee that the pigeons would not associate the testing stimuli with nonreinforcement and cease responding to them.

Test 2. In the next test, we continued to present Pair S3 versus S4, and we added two new testing pairs: Pair S1 versus S5 and Pair S2 versus S6. As in Test 1, the testing sessions included nine blocks, with the first block comprising 16 warm-up training trials. The next eight blocks contained 20 training trials and 3 testing trials, so that the pigeons received four presentations of each possible combination of the testing pairs. Thus, each session comprised 200 trials. Testing lasted for 10 days, in order for the pigeons to receive 40 ex- 


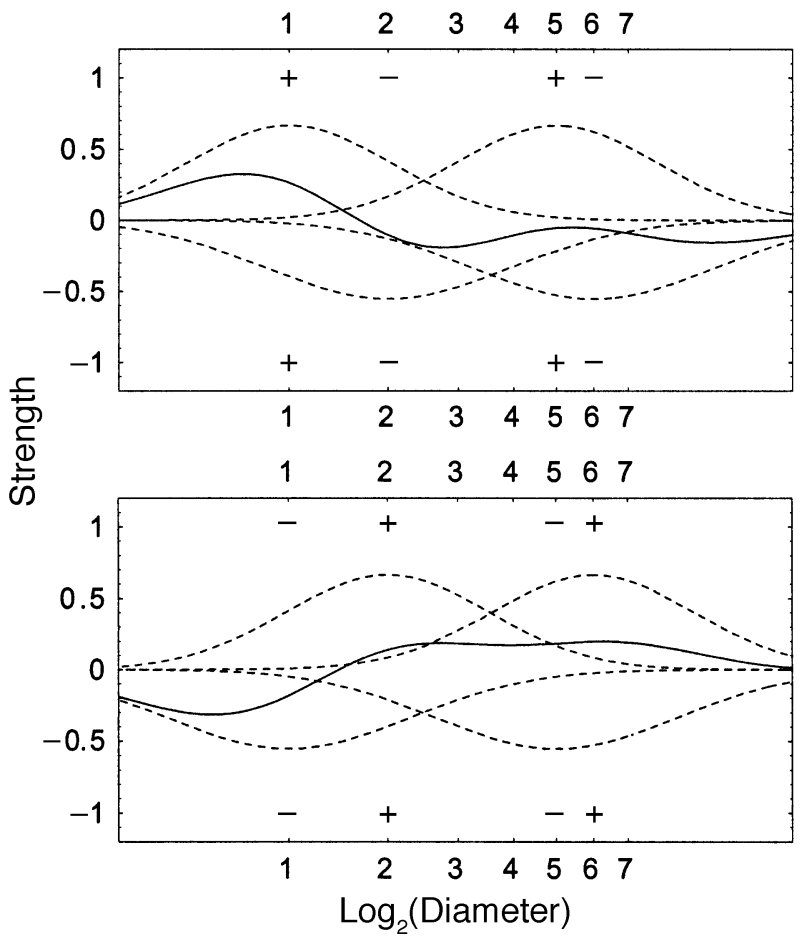

Figure 5. Sample postdiscrimination gradients (smooth lines) that predict near chance responding to Testing Pair S3 versus S4. The excitatory and inhibitory gradients are shown by dashed lines. Note that the postdiscrimination gradients fail to predict strong discrimination performance to Training Pair S5 versus S6 for both Smaller+ and Larger+ groups.

posures to each left-right combination of the testing stimuli: S3-S4 and $\mathrm{S} 4-\mathrm{S} 3, \mathrm{~S} 1-\mathrm{S} 5$ and $\mathrm{S} 5-\mathrm{S} 1$, and $\mathrm{S} 2-\mathrm{S} 6$ and S6-S2.

Test 3. In the final test, we presented Pair S3 versus Pair S4, Pair S1 versus S5, and Pair S2 versus S6, plus two novel testing pairs: Pair S2 versus S3 and Pair S4 versus S5. As in prior tests, the testing sessions comprised nine blocks, with the first block containing 16 warm-up training trials. The next eight blocks contained 20 training trials and 5 testing trials, so that the pigeons received four presentations of each possible combination of testing pairs. Thus, each session contained 216 trials. Testing lasted for 10 days, in order for the pigeons to receive 40 exposures to each left-right combination of the testing stimuli: S3-S4 and S4-S3, S1-S5 and S5-S1, S2-S6 and S6-S2, S2-S3 and S3-S2, and S4-S5 and S5-S4; therefore, testing yielded a total of 80 trials with each of the five testing pairs.

\section{Results and Discussion}

Training took a mean of 26.3 sessions, with a minimum of 15 sessions and a maximum of 52 sessions. Figure 6 shows the results averaged across all three tests, because no statistically significant changes in responding were detected to stimulus pairs that were given in two or more of the tests. This and later figures depict relationally correct responses; for example, for Pair S3 versus S4, the Smaller+ birds' choice of S3 was considered to be correct, whereas the Larger + birds' choice of S4 was considered to be correct. Figure 6 also shows the relationally correct responses that were predicted by Spence's (1937) theory, using the stimulus generalization gradients illustrated in Figures $3 \mathrm{~A}$ and $4 \mathrm{~A}$ (see Table 1). In order to arrive at these predictions, we took the net response strength to each pair of training and testing stimuli and applied Luce's (1959) choice rule (see Appendix A, Equation A3).

Two-tailed binomial tests disclosed that mean accuracy on all of the training trials (S1 vs. S2 and S5 vs. S6) was reliably different from the chance score of $50 \%$ correct $(z>36.01, p<.0001)$. More important, mean accuracy to all of the testing pairs (S1 vs. S5, S2 vs. S3, S3 vs. S4, S4 vs. S5, and S2 vs. S6) was also reliably higher than chance $(z \geq 2.40, p<.05)$.

A two-way analysis of variance (ANOVA; group $\times$ pair) yielded a significant main effect of pair $[F(6,18)=$ $24.02, p<.0001$. Tukey follow-up testing showed that relational responding to the training and testing pairs was ordered: $(\mathrm{S} 3$ vs. S4) $=(\mathrm{S} 2$ vs. S3 $)=(\mathrm{S} 4$ vs. S5 $)<$ $(\mathrm{S} 5$ vs. S6) $<(\mathrm{S} 1$ vs. S5) $<(\mathrm{S} 1$ vs. S2) $=(\mathrm{S} 2$ vs. S6) . Interestingly, performance on Training Pair $\mathrm{S} 5$ versus $\mathrm{S} 6$ was reliably lower than performance on Testing Pairs S1 versus $\mathrm{S} 5$ and $\mathrm{S} 2$ versus $\mathrm{S} 6$. The ANOVA also yielded a significant main effect of group $[F(1,3)=13.25, p<$ $.0001]$, as well as a significant group $\times$ pair interaction $[F(6,18)=11.75, p<.0001]$. Because the postdiscrimination generalization gradients predicted different patterns of responding in the Smaller+ and the Larger+ groups, we planned to compare the performance of these groups. Planned orthogonal contrasts disclosed that the birds in the Smaller+ group responded more accurately to Pair S1 versus S5 $[t(9)=-5.41, p<.0001]$ and to Pair S3 versus $\mathrm{S} 4[t(9)=-4.01, p<.0001]$ than did the birds in the Larger + group; responding to the other testing pairs did not differ significantly.

The significant group effect and the significant group $\times$ pair interaction could speak in favor of Spence's (1937) theory, since his gradient analysis yielded somewhat different predictions for the Smaller+ and the Larger+ groups. However, Figure 6 shows that the small behavioral differences to the training and the testing pairs in the Smaller+ and Larger+ groups did not accord with the large predicted differences, especially to Pair S2 versus S3 and to Pair S4 versus S5.

To summarize the results of Experiment 1, our pigeons exhibited reliable relational responding to all of the testing pairs, including S3 versus S4, although not at the same level of accuracy. This pattern of results was not predicted by any of the a priori gradient simulations. Still, no matter how carefully we selected the specific excitatory and inhibitory functions for those simulations, the objection can be raised that some other functions might fit our empirical data better; the realm of possible excitatory and inhibitory stimulus generalization gradients is vast indeed. So, we conducted a series of post hoc simulations, using Shepard's (1987) stimulus generalization function. ${ }^{1}$

Shepard (1987) has shown that a wide range of similarity judgments can be modeled using the Minkowski metric; this metric creates a symmetrical generalization function that monotonically decreases as a function of 


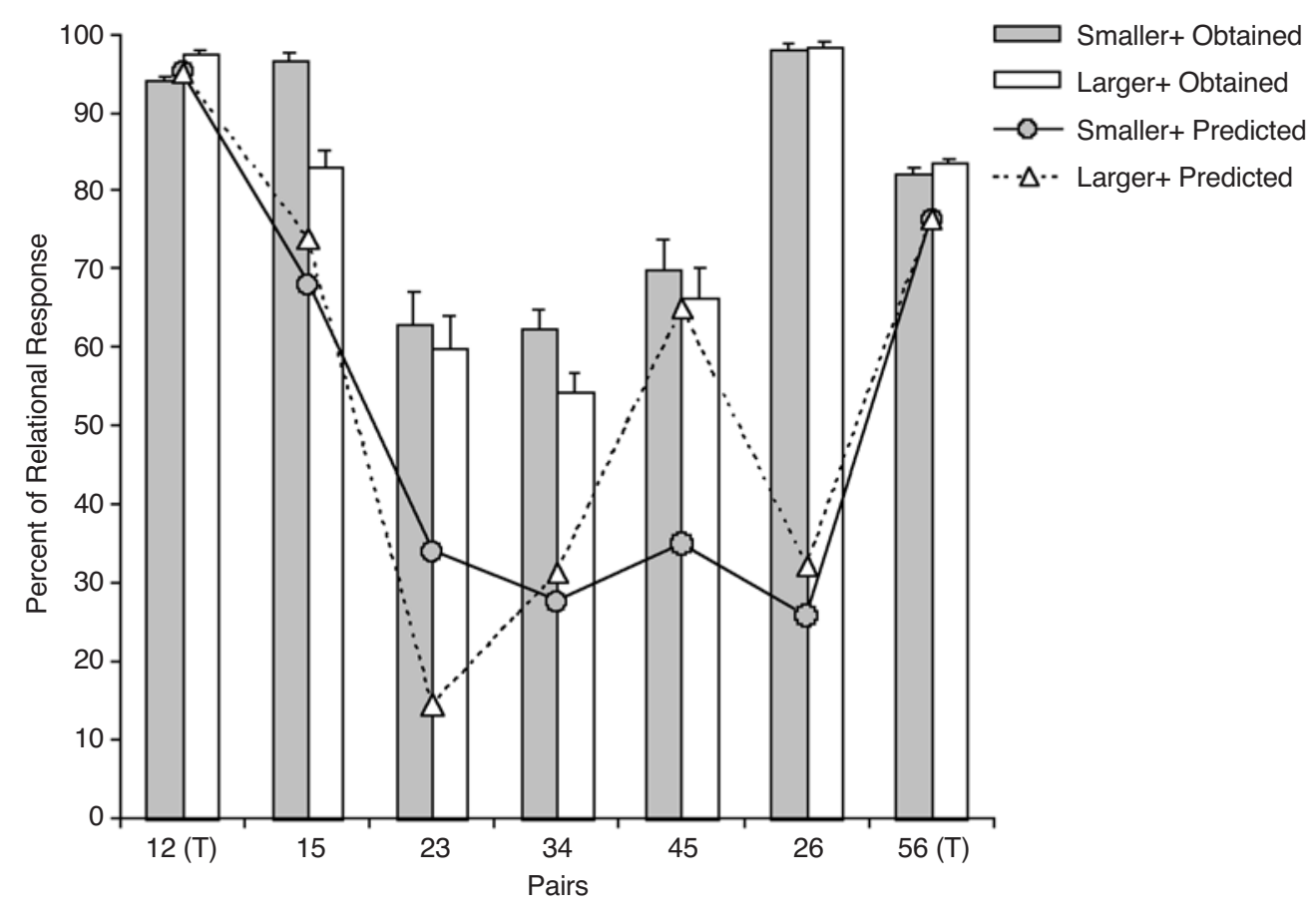

Figure 6. Percentages of relational responding across all three tests in the Smaller + and Larger + groups, as well as predictions obtained from the postdiscrimination gradients shown in Figures 3A and 4A (Experiment 1). The percentage of relational responding was significantly different from chance in all cases.

the similarity between the training and the testing items. The Gaussian function used in our simulations of Spence's (1937) theory (cf. Figure 1) is but one member of the broader class of functions proposed by Shepard. In addition, Shepard's stimulus generalization function has been shown to nicely fit empirical stimulus generalization data obtained from different species, including pigeons, and different stimulus domains, including circle size (Cheng, 1999; Cheng \& Spetch, 2002; Cheng, Spetch, \& Johnston, 1997; Shepard, 1987). A detailed description of Shepard's generalization model is provided in Appendix A.

We selected the best-fitting excitatory and inhibitory stimulus generalization gradients by fitting all of the training and testing scores, using the least-square method (see Appendix A for details). We also had to decide whether or not to adjust the bias parameter of Luce's (1959) choice rule; if a change in the bias parameter produced a better fit to the experimental data than the unbiased function, we saved those results. Importantly, any change in bias did not change the underlying stimulus generalization gradients; it only raised or lowered the predicted levels of choice responding.

Figure 7 shows the best-fitting excitatory and inhibitory stimulus generalization gradients. The upper row illustrates the predicted and obtained probabilities of pigeon's relational choice responding in the Smaller+ and Larger+ groups; in each case, the dotted line represents the unbiased predicted function, the dashed line represents the biased predicted function, and the solid line represents the empirical function. The second row portrays the un- derlying excitatory and inhibitory stimulus generalization gradients aligned vertically for easy comparison of their steepness. The third row depicts the excitatory and inhibitory stimulus generalization gradients placed at their proper positions along the size dimension, along with the algebraically derived postdiscrimination gradients. Finally, the bottom row portrays the excitatory and inhibitory gradients for S5 versus S6 only, along with the derived postdiscrimination gradient for that training pair.

At first glance, the top row depicting predicted and obtained relational responding suggests that Shepard's (1987) generalization model worked reasonably well; the predicted probabilities of choice responding did generally accord with the obtained choice functions, especially when the bias term of Luce's (1959) choice rule was used to raise the unbiased functions (dashed line). However, the ability of a theoretical model to fit empirical data does not by itself confirm its validity (Roberts \& Pashler, 2000); in order to do so, we need to extract and to examine the shapes of the underlying excitatory and inhibitory stimulus generalization gradients.

The second row of Figure 7 reveals that the simulated inhibitory gradient was slightly but correctly broader than the simulated excitatory gradient for the Larger+ group; however, the simulated excitatory gradient was slightly but incorrectly broader than the simulated inhibitory gradient for the Smaller+ group. It is also obvious that, in each case, the simulated excitatory and inhibitory stimulus generalization gradients were far broader than those expected after prolonged discrimination training 
Smaller+
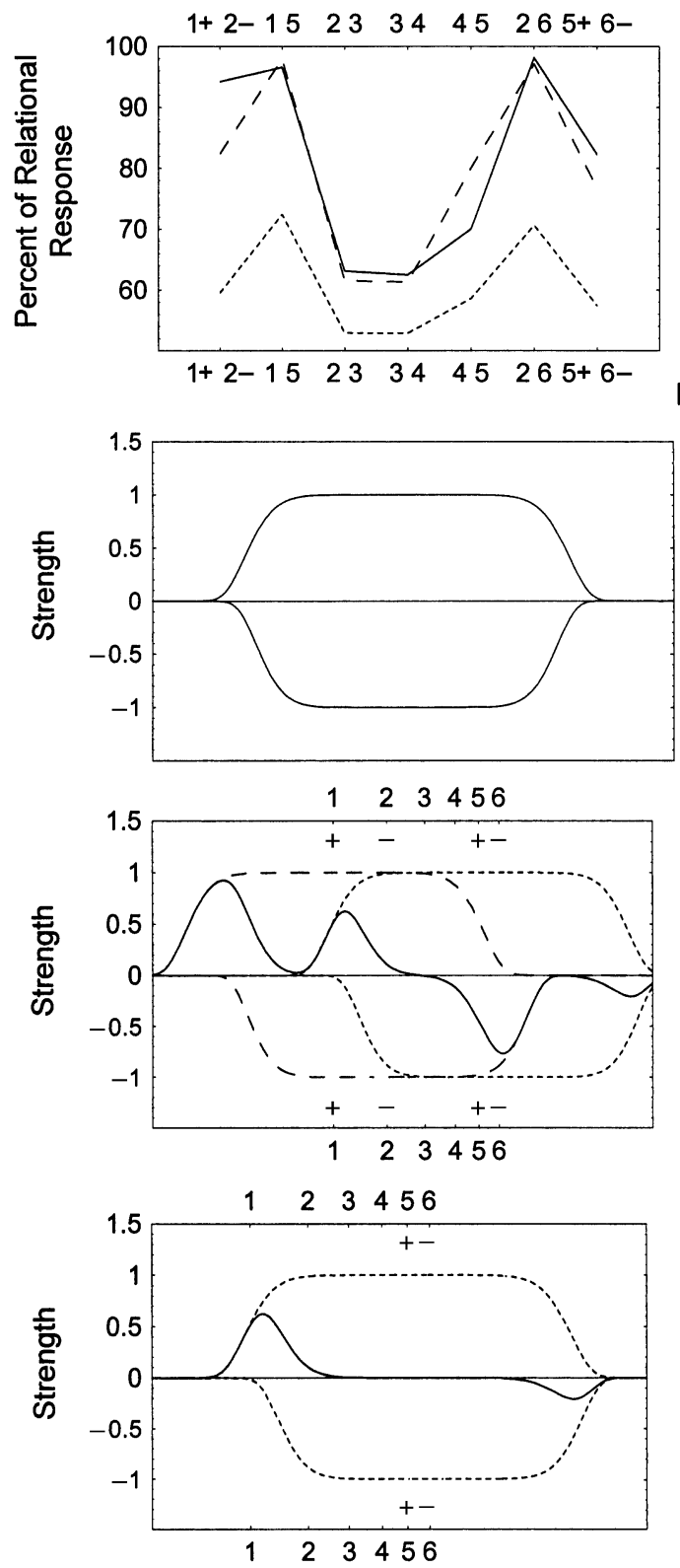

Larger+
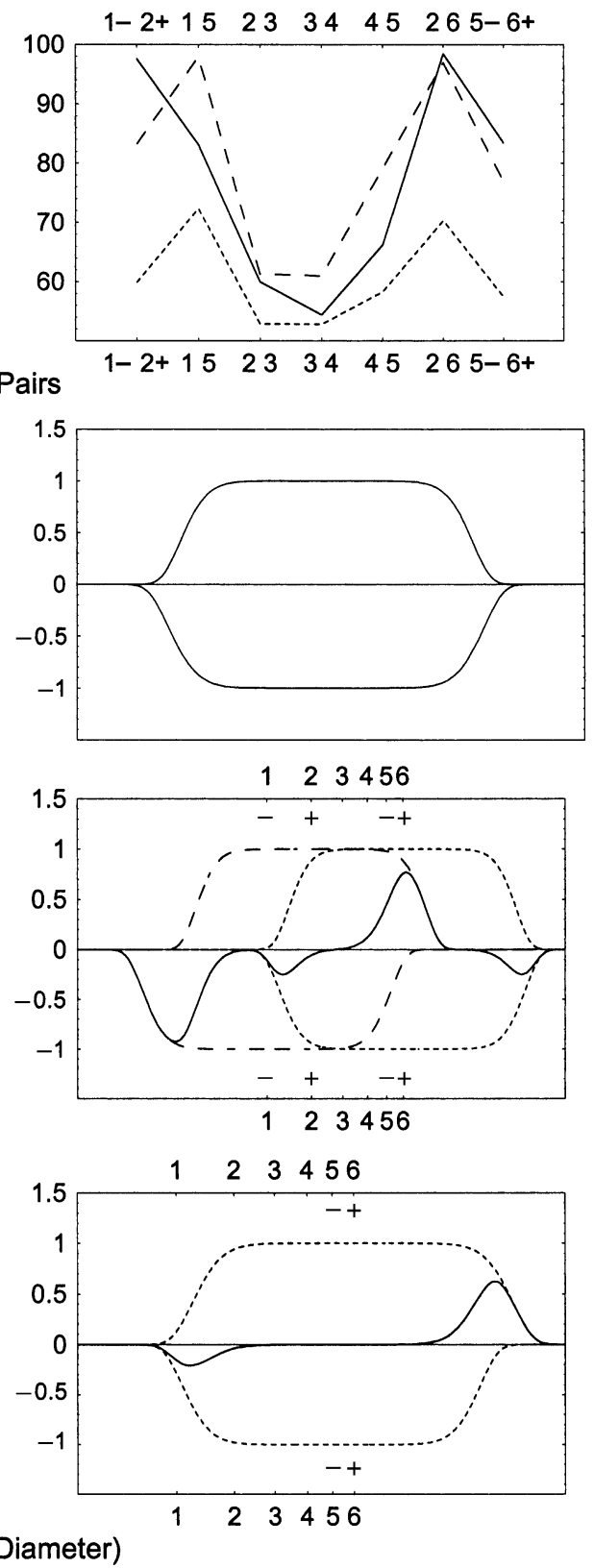

Figure 7. Best-fitting stimulus generalization gradients for the Smaller+ (left column) and Larger+ (right column) groups in Experiment 1, obtained with Shepard's model. The upper row shows experimental performance (smooth line), the original predicted values (dotted line), and the predicted values adjusted by a bias parameter (dashed line); the second row shows the individual excitatory and inhibitory gradients aligned for easy comparison; the third row shows the excitatory and inhibitory gradients (dashed lines) and the postdiscrimination gradients (smooth line); the bottom row shows the excitatory and inhibitory gradients in the case of one-pair, $\mathrm{S} 5$ versus $\mathrm{S} 6$ training. The best-fitting parameters for Smaller+ were $i=8.99, c_{\mathrm{E}}=0.01, c_{\mathrm{I}}=0.02$, and for Larger+ were $i=8.89, c_{\mathrm{E}}=0.02, c_{\mathrm{I}}=0.01$ (see Appendix A for notations).

(compare these gradients with the empirically inspired gradients depicted in Figure 1).

We suggested earlier that, for an absolute theory of discrimination learning to predict relational responding to the testing pairs, one must assume very broad generalization gradients, so that stimuli in the middle of the dimensional domain (S3 and S4) will receive substantial generalized associative strength (cf. Figure 5). The de- 
rived excitatory and inhibitory functions were certainly broad-probably unrealistically so. We also suggested that such broad stimulus generalization gradients ought to create considerable difficulty in predicting discrimination of the training pairs. Surprisingly, the upper row of Figure 7 suggests that Shepard's (1987) model did not have much difficulty in accounting for discriminative responding to the training pairs. Why not?

The answer to this question comes by considering the postdiscrimination stimulus generalization gradients (solid lines) depicted in the third row of Figure 7. These gradients were generally monotonic from the smallest to the largest stimuli shown in training and testing. Such monotonic postdiscrimination gradients necessarily predict relational responding to all of the stimulus pairs of interest, assuming that the gradients fall after Smaller+ training and rise after Larger + training. But monotonic postdiscrimination gradients, such as those in Figure 7, entail a most improbable characteristic: Some reinforced training stimuli assume net inhibitory values (S5 in the Smaller+ group and $\mathrm{S} 2$ in the Larger+ group). Predicting relational responding across the full range of training and testing stimuli thus came with considerable theoretical costs: (1) unrealistically broad generalization gradients of excitation and inhibition and (2) S+ stimuli of net inhibitory strength.

A further cost associated with our simulation of Shepard's (1987) generalization theory is its failure to make plausible predictions of discrimination learning from the derived excitatory and inhibitory gradients arrived at from all of the training and testing pairs. Assuming that the derived gradients in the second row of Figure 7 are representative of training around single $\mathrm{S}+$ and $\mathrm{S}-$ stimuli, we applied these gradients to the simple case of onepair discrimination training between S5 and S6; these results are illustrated in the bottom row of Figure 7 (the results of one-pair training between S1 and S2 are essentially the same and thus are not shown). It is obvious that these gradients do not support discrimination learning between $\mathrm{S} 5+$ and $\mathrm{S} 6-$ or between S5- and S6+; all of the associative values of these $\mathrm{S}+$ and $\mathrm{S}-$ stimuli are zero. Thus, these excitatory and inhibitory stimulus generalization gradients cannot plausibly serve as the associative substrate for relational responding with multiplepair discrimination training, unless it is assumed that gradients of excitation and inhibition around the $\mathrm{S}+$ and $\mathrm{S}$ - stimuli differ depending on the number and location of other S+ and S- stimuli given at other times during training. Spence (1937) did not make this assumption, since it would have violated the very tenets of his elegant associative model.

Thus, these many post hoc simulation failures, coupled with the many earlier a priori simulation failures, strongly suggest that a discrimination theory of absolute responding simply cannot predict the relational responding that we observed in our two-pair discrimination-learning experiment. In that experiment, our pigeons responded relationally to all five testing pairs. Interestingly, the strength of relational responding differed from pair to pair (Figure 6). We observed the lowest levels of relational responding to those testing pairs that contained at least one stimulus that had never been presented before (S3 vs. S4, S2 vs. $\mathrm{S} 3$, and S4 vs. S5); the testing pairs that controlled the highest levels of relational responding entailed previously trained stimuli (S1 vs. S5 and S2 vs. S6). The simplest explanation for these results is that testing performance involving S3 and S4 was impaired by the sheer novelty of these cues. So, in Experiment 2, we decided to address this explanation, while exposing Spence's (1937) theory to an even stricter empirical test.

\section{EXPERIMENT 2}

We designed the multiple discrimination training task in Experiment 2 so that pigeons would receive exposure to all six of the training and testing stimuli but absolute and relational accounts of discrimination learning and transfer would yield different behavioral predictions. Here, the birds were given four discriminations: S1 versus S2, S1 versus S3, S4 versus S6, and S5 versus S6. Both Smaller + and Larger + tasks were arranged. Now, the birds would gain familiarity with S3 and S4 during training. The critical test was again S3 versus S4. As in Experiment 1, relational responding in the Smaller+ group would lead to the choice of S3 over S4 despite the fact that, in original discrimination learning, $\mathrm{S} 3$ had been an $\mathrm{S}-$ and $\mathrm{S} 4$ had been an $\mathrm{S}+$; relational responding in the Larger+ group would lead to the choice of S4 over $\mathrm{S} 3$ despite the fact that, in original discrimination learning, $\mathrm{S} 4$ had been an $\mathrm{S}-$ and $\mathrm{S} 5$ had been an $\mathrm{S}+$. Also as in Experiment 1, we tested the pigeons with other untrained stimulus pairings that could effectively discriminate absolute from relational responding.

\section{Simulations}

Just as we did prior to Experiment 1, we again conducted similarly designed a priori simulations of four-pair discrimination training, using the same excitatory and inhibitory stimulus generalization gradients. Figure 8 illustrates these simulations for the Smaller+ and Larger+ groups. ${ }^{2}$ Figure 9 depicts the mean percentages of relational choices to all of the training and testing pairs predicted by the stimulus generalization gradients in Figure 8.

The interacting excitatory and inhibitory stimulus generalization gradients yielded different predictions for the Smaller+ and the Larger+ groups. Unlike Experiment 1 , the net postdiscrimination gradients could now predict relational responding both to $\mathrm{S} 1$ versus $\mathrm{S} 5$ and to $\mathrm{S} 4$ versus S5 (cf. Table 1). However, these gradients also predicted absolute responding to $\mathrm{S} 3$ versus $\mathrm{S} 4$, an unsurprising outcome, given the fact that, during training, one of the stimuli was directly reinforced and the other was directly nonreinforced. Testing Pair S2 versus S3 also posed a problem for Spence's (1937) theory, which predicted absolute, rather than relational, responding. 


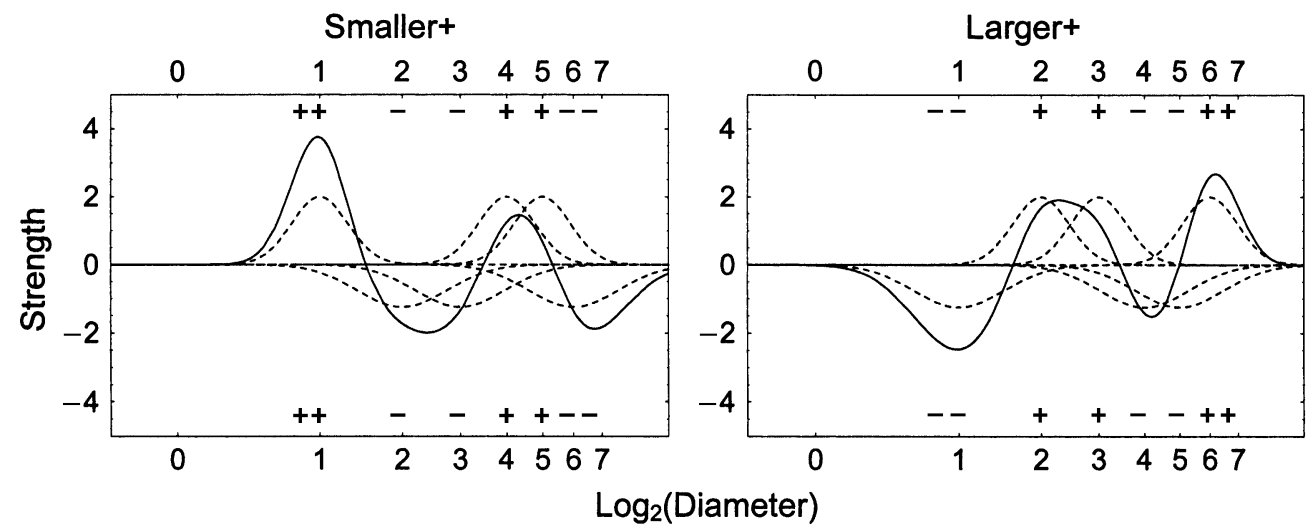

Figure 8. Postdiscrimination gradients (smooth lines) obtained by summation of the excitatory and the inhibitory gradients (dashed lines) for birds trained to discriminate S1 versus S2, S1 versus S3, S4 versus S5, and S5 versus S6 (Experiment 2).

\section{Method}

\section{Subjects}

The subjects were 4 different feral pigeons housed and maintained as in Experiment 1. Prior to the present experiment, the pigeons had served in unrelated projects.

\section{Apparatus and Stimuli}

The same apparatus and stimuli as those in Experiment 1 were used.

\section{Design}

As in Experiment 1, the pigeons were trained to discriminate the two end pairs of circles: $\mathrm{S} 1$ versus $\mathrm{S} 2$ and $\mathrm{S} 5$ versus S6. In addition, they also received training with two other pairs of circles: S1 versus $\mathrm{S} 3$ and $\mathrm{S} 4$ versus $\mathrm{S} 6$. The 4 pigeons were randomly assigned to the Smaller+ group $(n=2)$ and to the Larger+ group $(n=2)$. For the Smaller+ group, the smaller of two simultaneously presented circles was always correct; thus, their training can be denoted $\mathrm{S} 1+$ versus $\mathrm{S} 2-, \mathrm{S} 1+$ versus $\mathrm{S} 3-, \mathrm{S} 4+$ versus $\mathrm{S} 6-$, and $\mathrm{S} 5+$ versus $\mathrm{S} 6-$. For the Larger+ group, the larger of two simultaneously presented circles was always correct; thus, their training can be denoted $\mathrm{S} 1-$ versus $\mathrm{S} 2+, \mathrm{S} 1-$ versus $\mathrm{S} 3+, \mathrm{S} 4-$ versus $\mathrm{S} 6+$, and $\mathrm{S} 5-$ versus $\mathrm{S} 6+$. After training, the pigeons were tested with the same five novel pairs of stimuli as those in Experiment 1: S1 versus $\mathrm{S} 5, \mathrm{~S} 2$ versus $\mathrm{S} 3, \mathrm{~S} 3$ versus $\mathrm{S} 4, \mathrm{~S} 4$ versus $\mathrm{S} 5$, and $\mathrm{S} 2$ versus $\mathrm{S} 6$.

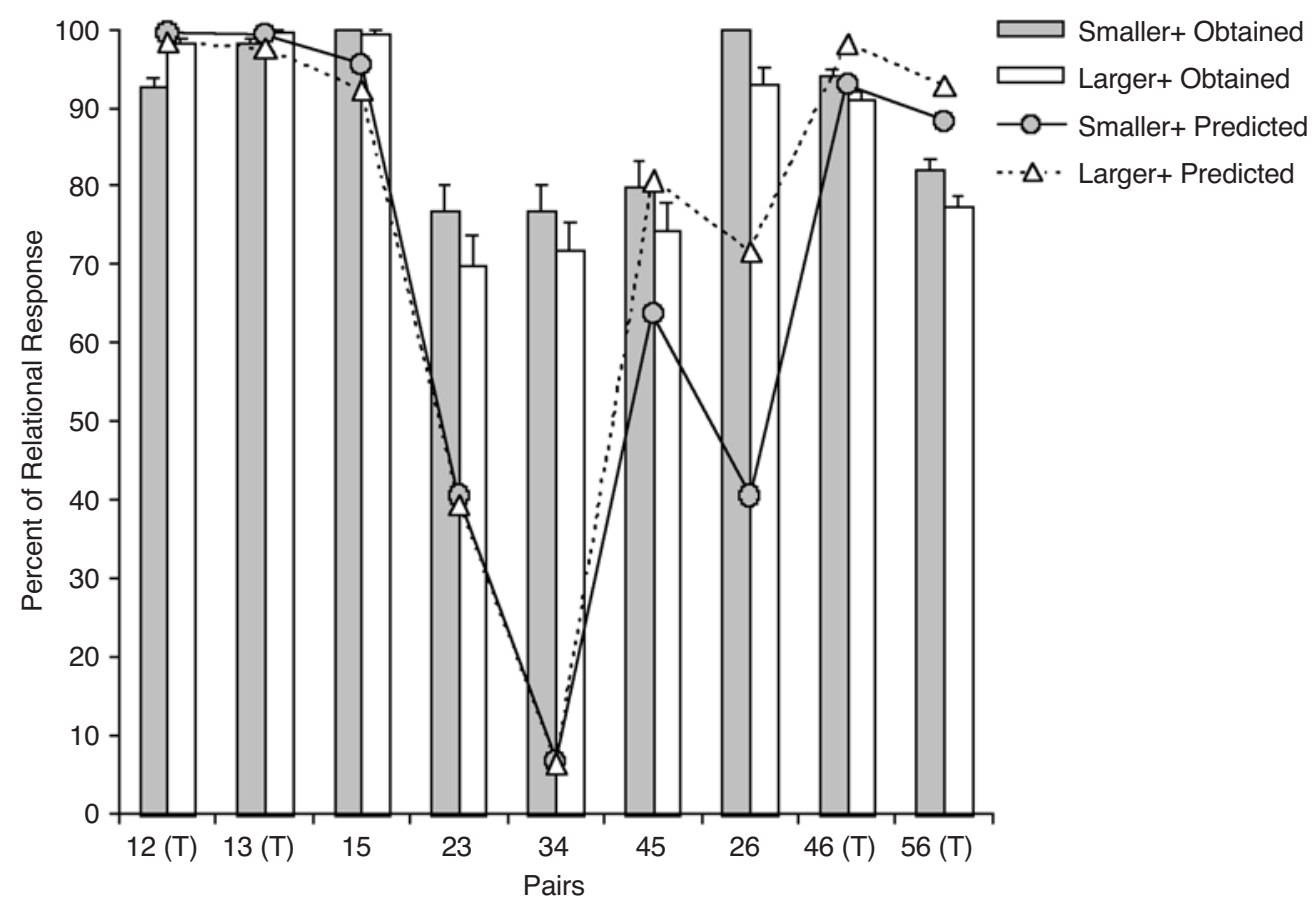

Figure 9. Percentages of relational responding across all three tests in the Smaller+ and Larger+ groups, as well as predictions obtained from the postdiscrimination gradients shown in Figure 8 (Experiment 2). The percentage of relational responding was significantly different from chance in all cases. 


\begin{abstract}
Procedure
Pretraining and training. The same pretraining and training procedures as those in Experiment 1 were used, with due allowance for the extra training stimuli. During training, each session comprised 200 trials involving 25 blocks of 8 trials; the left-right locations of correct responding were randomized within each block. All the birds had to meet an $80 / 75$ criterion $(80 \%$ correct overall and $75 \%$ correct to each of the eight possible stimulus combinations); we lowered the criterion to accommodate twice as many stimulus combinations here as in Experiment 1. The discrimination criterion had to be maintained during testing; if a bird's discrimination performance fell below criterion, it was returned to training until it again reached criterion.

Testing. As in Experiment 1, in Test 1, only the middle pair composed of S3 and S4 was presented. In Test 2, we continued to present Pair S3 versus S4, plus we added two new pairs: Pair S1 versus S5 and Pair S2 versus S6. In Test 3, we presented Pair S3 versus S4, Pair S1 versus S5, and Pair S2 versus S6, plus two novel testing pairs: Pair S2 versus S3 and Pair S4 versus S5. Each test lasted for 10 sessions, so the birds would receive 40 exposures to each left-right combination of the testing stimuli (a total of 80 trials with each of the five testing pairs).
\end{abstract}

\section{Results and Discussion}

Training took a mean of 33.8 sessions, with a minimum of 26 sessions and a maximum of 43 sessions. Figure 9 shows the mean percentages of relational choices to all of the training and testing pairs, as well as performance predicted by the stimulus generalization gradients illustrated in Figure 8 (after fitting predicted strength to the data, using Luce's [1959] rule).

Two-tailed binomial tests disclosed that mean accuracy to all of the training and testing pairs was significantly above chance $(z \geq 3.3, p<.05)$. A two-way ANOVA (group $\times$ pair) revealed a significant main effect of pair $[F(8,24)=289.56, p<.0001]$. Given the results of Experiment 1, we expected relational responding to Pair S3 versus $\mathrm{S} 4$ to be lowest, responding to Pairs S1 versus S5 and $\mathrm{S} 2$ versus $\mathrm{S} 6$ to be highest, and responding to the remaining testing pairs to be intermediate. Planned comparisons supported these expectations. Responding to the stimuli was ordered: $(\mathrm{S} 3$ vs. $\mathrm{S} 4)=(\mathrm{S} 2$ vs. $\mathrm{S} 3)=(\mathrm{S} 4$ vs. S5 $)=(\mathrm{S} 5$ vs. S6) $<(\mathrm{S} 4$ vs. S6) $<(\mathrm{S} 1$ vs. S2 $)=(\mathrm{S} 2$ vs. S6) $=(\mathrm{S} 1$ vs. S3) $=(\mathrm{S} 1$ vs. S5). Similar to Experiment 1, responding to Training Pairs S5 versus S6 and S4 versus S6 was significantly lower than that to Testing Pairs S1 versus S5 and S2 versus S6.

The ANOVA also revealed a significant group main effect $[F(1,3)=15.43, p<.001]$, as well as a significant pair $\times$ group interaction $[F(8,24)=12.23, p<.001]$. Planned orthogonal contrasts showed that the Smaller+ group responded significantly more accurately to Pairs $\mathrm{S} 2$ versus $\mathrm{S} 3, \mathrm{~S} 3$ versus $\mathrm{S} 4$, and $\mathrm{S} 2$ versus $\mathrm{S} 6(t \leq-2.24$, $p<.05)$. Note that gradient theory predicted no difference in relational responding to Pairs S2 versus S3 and $\mathrm{S} 3$ versus $\mathrm{S} 4$, and it predicted more accurate relational responding in the Larger+ group to Pair S2 versus S6.

In short, we again observed a high level of relational responding to all of the testing pairs. Note that, in this experiment, S3 and S4 had reinforcement histories that should have strongly opposed relational responding. For instance, pigeons in the Smaller + group were exposed to $\mathrm{S} 1+$ versus $\mathrm{S} 3-$ and to $\mathrm{S} 4+$ versus $\mathrm{S} 6-$. If the birds were to respond relationally, they had to select previously nonreinforced S3 and to reject previously reinforced S4. If anything, Spence's (1937) theory of absolute responding predicts that relational responding to S3 versus S4 in Experiment 2 ought to have been even lower than that in Experiment 1, where S3 and S4 could receive only generalized excitation or inhibition because they were not explicitly trained.

So, we conducted a full-factorial ANOVA with experiment, group, and testing pair as factors. All three of the main effects were statistically significant $(F \geq 24.42$, $p<.0001)$. The ANOVA also revealed a significant experiment $\times$ pair interaction $[F(4,28)=7.45, p<.0001]$. Tukey follow-ups disclosed that responding to Testing Pairs S2 versus S6 and S4 versus S5 did not differ significantly between experiments; but responding to Testing Pairs S1 versus S5, S2 versus S3, and S3 versus S4, was significantly higher in Experiment 2 than in Experiment 1, contrary to Spence's (1937) theory. ${ }^{3}$

This notable enhancement in relational responding led us to suspect that the two added training pairs instantiating the same stimulus relation may have done more than simply acquaint the pigeons with S3 and S4. It is well known that increasing the number of exemplars leads to better acquisition of a concept in many species of animals, including pigeons (Cook, 2002; Katz, Wright, \& Bachevalier, 2002; Wright, 1997; Wright, Cook, Rivera, Sands, \& Delius, 1988); the same might be true for learning the relation between or among stimuli along a dimension. This issue was addressed in our third experiment.

\section{EXPERIMENT 3}

To see if four-pair discrimination training had actually increased the generality of relational responding, we added two new testing stimuli in Experiment 3: the smallest, S0, with a diameter of $0.35 \mathrm{~cm}$, and the largest, S7, with a diameter of $2.87 \mathrm{~cm}$. In testing, we presented Pairs S0 versus S7, S0 versus S4, and S3 versus S7 to 2 of the pigeons that had just completed Experiment 2. If discrimination training in Experiment 2 with S3 versus S4 had merely eliminated the novelty of these stimuli when they were given in Experiment 1, we might expect the pigeons in Experiment 3 to respond at only a moderate level of relational accuracy to Pairs S0 versus S7, S0 versus S4, and S3 versus S7, much as the pigeons in Experiment 1 had responded to Pairs S3 versus S4, S2 versus $\mathrm{S} 3$, and S4 versus S5, because all of these choice tests involved novel stimuli. On the other hand, if four-pair discrimination training had actually increased the generality of the pigeons' relational responding, we might expect the pigeons in Experiment 3 to exhibit a high level of relational responding to Pairs $\mathrm{S} 0$ versus $\mathrm{S} 7, \mathrm{~S} 0$ versus S4, and $\mathrm{S} 3$ versus $\mathrm{S} 7$, perhaps comparable to their prior performance to Pairs S1 versus S5 and S2 versus S6. 


\section{Simulations}

Figure 8 shows the simulations for the Smaller+ and the Larger+ groups of Experiment 3 (note that this figure also includes the two new stimuli: S0 and S7); Figure 10 depicts the pattern of responding predicted by the interacting gradients shown in Figure 8. Again, the stimulus generalization gradients of excitation and inhibition that we explored could not account for relational responding to all of these stimulus pairs at the same time. Particularly troublesome for Spence's (1937) stimulus generalization theory was newly added Testing Pair S0 versus S4.

\section{Method}

\section{Subjects}

Two pigeons from Experiment 2 were studied; 1 received Smaller+ training, and 1 received Larger + training. The birds were housed and maintained as in Experiments 1 and 2.

\section{Stimuli and Apparatus}

The same apparatus and stimuli as those in Experiments 1 and 2 were used. Two additional stimuli were created: one a circle of $0.35-\mathrm{cm}$ diameter $(\mathrm{S} 0)$ and the other a circle of $2.87-\mathrm{cm}$ diameter (S7).

\section{Training and Testing}

In order to proceed to testing, the pigeons were again required to meet the $80 / 75$ criterion $(80 \%$ correct overall and $75 \%$ correct to each of the eight possible combinations of stimuli). The criterion had to be maintained during testing; if a bird's discrimination performance fell below criterion, it was returned to training until it again reached criterion. In testing, we presented Testing Pair S3 versus S4 plus three new testing pairs: $\mathrm{S} 0$ versus $\mathrm{S} 7, \mathrm{~S} 0$ versus $\mathrm{S} 4$, and $\mathrm{S} 3$ versus $\mathrm{S} 7$. This test lasted for 10 sessions, so that the pigeons would receive 40 exposures to each left-right combination of the testing stimuli (a total of 80 trials with each of the three testing pairs).

\section{Results and Discussion}

Figure 10 illustrates the relational responding of each pigeon to the new testing pairs ( $\mathrm{S} 0$ vs. S7, S0 vs. S4, and $\mathrm{S} 3$ vs. S7) and to the familiar training pairs in Experiment 3 , along with their responding during testing in Experiment 2 to the prior testing pairs (S1 vs. S5, S2 vs. S3, $\mathrm{S} 3$ vs. S4, S4 vs. S5, and S2 vs. S6). Figure 10 also depicts the pattern of responding that is predicted by the interacting stimulus generalization gradients shown in Figure 8.

Two-tailed binomial tests revealed that mean relational responding to all of the training and testing pairs in Experiment 3 was significantly above chance $(z \geq 3.3$, $p<.05)$. Contrary to the predictions derived from the postdiscrimination stimulus generalization gradients, both birds responded perfectly relationally to all three of the novel testing pairs.

Such a high level of relational responding to the testing stimuli- two of which (S0 and S7) were smaller than and larger than, respectively, any other stimuli seen beforesuggests that four-pair discrimination training really did increase the generality of relational responding, as compared with two-pair training. It is hardly surprising, therefore, that studies in which pigeons were trained with only one pair per dimension showed very little evidence of relational responding (e.g., Wills \& Mackintosh, 1999).

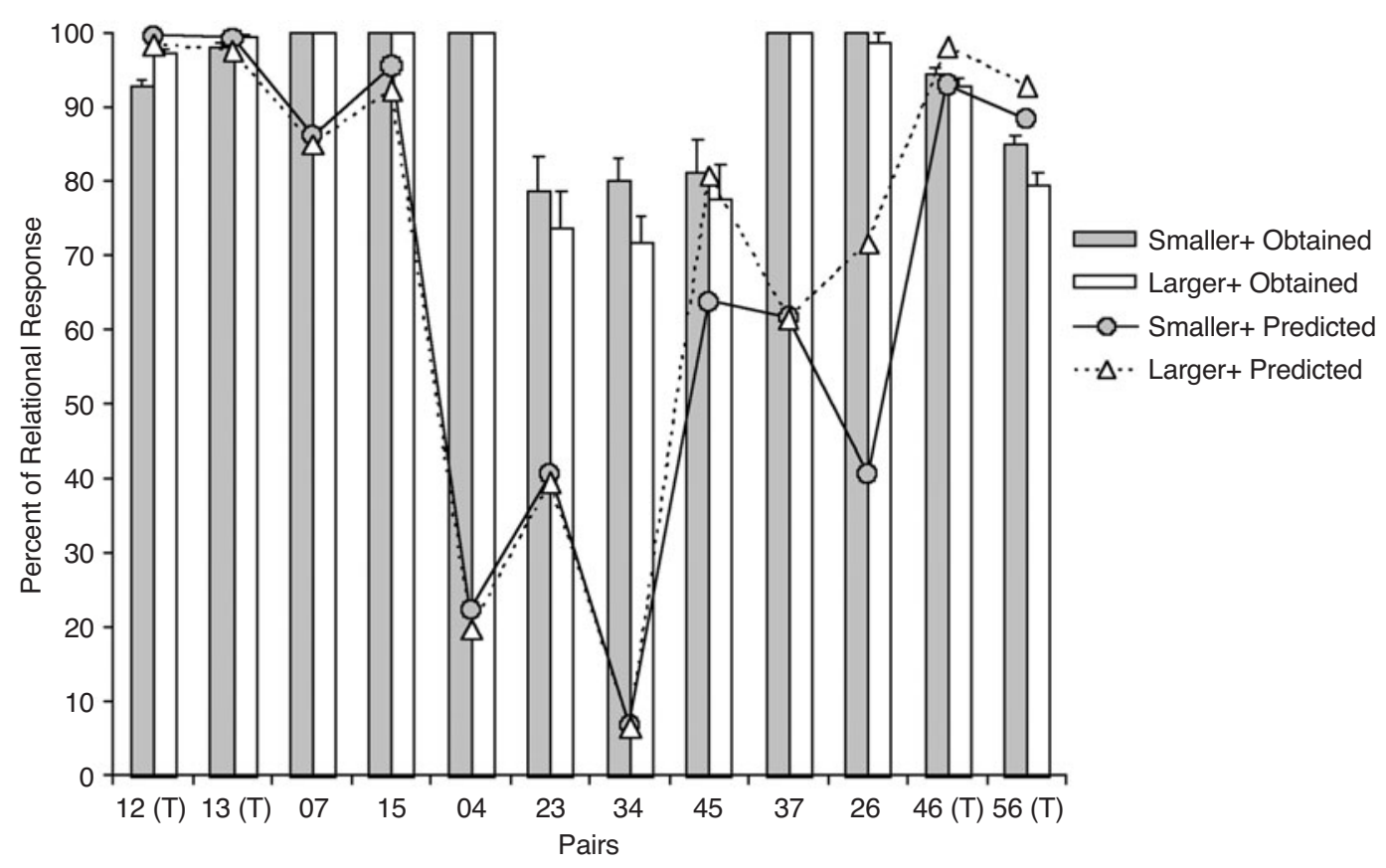

Figure 10. Percentages of relational responding to the testing pairs in Experiment 3 (S0 vs. S7, S0 vs. S4, S3 vs. S7) and to the prior testing pairs (Experiment 2: S1 vs. S5, S2 vs. S3, S3 vs. S4, and S4 vs. S5) as well as predictions obtained from the postdiscrimination gradients shown in Figure 8 (Experiment 3). The percentage of relational responding was significantly different from chance in all cases. 
Apparently, just as in the case of concept learning, pigeons need to encounter multiple instances of a rule in order to exhibit strong relational responding.

\section{EXPERIMENT 4}

In all of our prior experiments, we gave pigeons simultaneous multiple-pair discrimination training. However, many earlier reports used successive one-pair discrimination training. It might be that multiple-pair discrimination training is a necessary and sufficient condition to obtain relational responding. We would thus expect that if our pigeons were exposed to simultaneous one-pair training, they should respond exactly as predicted by Spence's (1937) theory. This idea was explored in our fourth and final experiment.

In this experiment, each of 4 pigeons received exposure to only one of the four possible training pairs given to the pigeons in Experiment 1. The same five testing pairs as those in Experiments 1 and 2 were given: $\mathrm{S} 1$ versus $\mathrm{S} 5, \mathrm{~S} 2$ versus $\mathrm{S} 3, \mathrm{~S} 3$ versus $\mathrm{S} 4, \mathrm{~S} 4$ versus $\mathrm{S} 5$, and $\mathrm{S} 2$ versus $\mathrm{S} 6$. In addition, the pigeons were given three testing pairs from Experiment 3 (S0 vs. S7, S0 vs. S4, and S3 vs. S7), plus the remaining (untrained) testing pair (either S1 vs. S2 or S5 vs. S6).

\section{Simulations}

The a priori simulations for one-pair discrimination training, using the same excitatory and inhibitory stimulus generalization gradients as those in Experiments 1, 2, and 3, are illustrated in Figure 2. Table 2 summarizes the patterns of relational responding derived from those gradients. The interacting excitatory and inhibitory stimulus generalization gradients yielded different predictions for the different training regimes, with slightly more absolute responding after $\mathrm{S} 1$ versus $\mathrm{S} 2$ training than after $\mathrm{S} 5$ versus S6 training. In neither case, however, did the majority of testing pairs support robust relational responding. ${ }^{4}$

\section{Method}

\section{Subjects}

The subjects were 4 different feral pigeons housed and maintained as in Experiment 1. Prior to the present experiment, the birds had served in unrelated experiments.

\section{Apparatus and Stimuli}

The same apparatus and stimuli as those in Experiment 2 and 3 were used.

\section{Design}

The 4 pigeons were randomly assigned to either S1 versus S2 $(n=2)$ training or to S5 versus S6 $(n=2)$ training. Within each group, the pigeons were assigned to the Smaller+ group or to the Larger + group. After discrimination mastery, the pigeons were tested with the same five novel pairs of stimuli as in Experiment 2: $\mathrm{S} 1$ versus $\mathrm{S} 5, \mathrm{~S} 2$ versus $\mathrm{S} 3, \mathrm{~S} 3$ versus $\mathrm{S} 4, \mathrm{~S} 4$ versus $\mathrm{S} 5$, and $\mathrm{S} 2$ versus $\mathrm{S} 6$. In the second test, they were tested with the same additional stimulus pairs as in Experiment 3-S0 versus S7, S0 versus S4, and $\mathrm{S} 3$ versus $\mathrm{S} 7$ - as well as with either S1 versus S2 (for S5 vs. S6 training) or to S5 versus S6 (for S1 vs. S2 training).

After these tests were completed, the pigeons were given two-pair discrimination training with S1 versus S2 and with S5 versus S6, in order to see whether expanding the number of training pairs would enhance the birds' relational responding. The pigeons were then tested with the same five stimulus pairs as in Experiment 2: $\mathrm{S} 1$ versus $\mathrm{S} 5, \mathrm{~S} 2$ versus $\mathrm{S} 3, \mathrm{~S} 3$ versus $\mathrm{S} 4, \mathrm{~S} 4$ versus $\mathrm{S} 5$, and $\mathrm{S} 2$ versus $\mathrm{S} 6$.

\section{Procedure}

Pretraining and training. The same pretraining and training procedures as those in Experiment 1 were followed, with small changes to accommodate one-pair training. During training, each session comprised 200 trials involving 25 blocks of 8 trials; the left-right locations of the correct stimulus were randomized within each block. All the birds had to meet the $80 \%$ correct criterion before proceeding to the test. This criterion had to be maintained during testing; if a bird's discrimination performance fell below criterion, it was returned to training until it again reached criterion.

Testing. Because neither Experiment 1 nor Experiment 2 yielded any differences in choice responding during Tests 1,2, or 3, we first conducted Test 3 , which included all five testing pairs. In this test, we presented the training pair, plus five novel testing pairs: S3 versus $\mathrm{S} 4, \mathrm{~S} 1$ versus S5, S2 versus S6, S2 versus S3, and S4 versus S5. In the next test, we presented the three testing pairs from Experiment 3 (S0 vs. S7, S0 vs. S4, and S3 vs. S7), plus the remaining (untrained) pair (S1 vs. S2 or S5 vs. S6). Each test lasted 10 sessions, in order for the birds to receive 40 exposures to each left-right combination of the testing stimuli (a total of 80 trials with each of the testing pairs).

Two-pair training and testing. The same training procedure as that in Experiment 1 was followed. Only Test 3, which included all five testing pairs, was conducted.

\section{Results and Discussion}

\section{One-Pair Training and Testing}

Training took a mean of 11 sessions, with a minimum of 6 sessions and a maximum of 20 sessions. Figure 11

Table 2

Percentages of Relational Responding to the Training and Testing Pairs After One-Pair Discrimination Training (Experiment 4) Obtained From Simulated Postdiscrimination Gradients, Using Luce's Choice Rule (Appendix A, Equation A3)

\begin{tabular}{|c|c|c|c|c|c|c|c|c|c|c|}
\hline Group & 1 vs. 2 & 0 vs. 7 & 1 vs. 5 & 0 vs. 4 & 2 vs. 3 & 3 vs. 4 & 4 vs. 5 & 3 vs. 7 & 2 vs. 6 & 5 vs. 6 \\
\hline \multicolumn{11}{|c|}{ S1 Versus S2 Training } \\
\hline Smaller + & 95.17 & 50.00 & 85.45 & 52.08 & 33.62 & 38.79 & 48.16 & 36.84 & 22.82 & 49.79 \\
\hline Larger+ & 95.17 & 50.19 & 77.19 & 50.23 & 17.69 & 44.47 & 49.96 & 44.43 & 14.67 & 50.00 \\
\hline \multicolumn{11}{|c|}{ S5 Versus S6 Training } \\
\hline Smaller + & 50.02 & 69.24 & 26.61 & 38.38 & 50.58 & 37.81 & 36.79 & 68.72 & 54.20 & 76.57 \\
\hline Larger+ & 49.76 & 66.06 & 45.77 & 31.19 & 44.43 & 36.39 & 65.06 & 71.07 & 73.58 & 76.59 \\
\hline
\end{tabular}

Note-Bold font denotes nonrelational choice, and italicized font denotes the training pairs. 

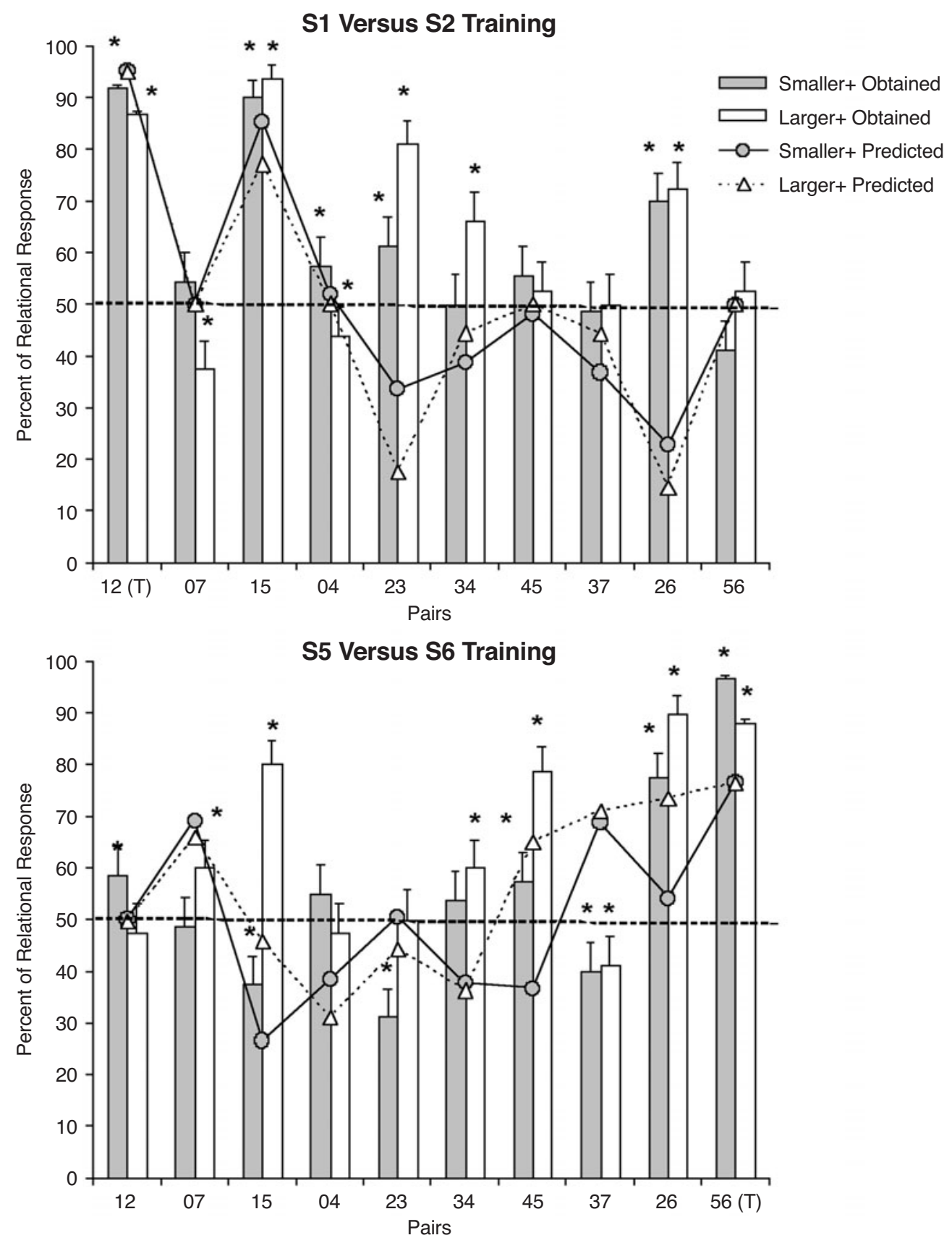

Figure 11. Percentages of relational responding after $S 1$ versus $S 2$ training (upper panel) and after $S 5$ versus S6 training (lower panel), as well as predictions obtained from the postdiscrimination gradients shown in Figure 2 (Experiment 4). Asterisks indicate that the percentage of relational responding was significantly different from chance.

shows the mean percentage of relational choices to all of the training and testing pairs for each of the 4 birds, as well as performance predicted by the stimulus generalization gradients shown in Figure 2.

Two-tailed binomial tests revealed that mean relational responding to all of the training pairs was significantly above chance level $(z \geq 3.3, p<.05)$. Responding to some of the testing pairs was also reliably above chance (e.g., S2 vs. S6, both panels); responding to other testing pairs was reliably below chance (e.g., S3 vs. S7, lower panel). Did these patterns of performance correspond with the predictions derived from the excitatory and inhibitory stimulus generalization gradients?

According to the postdiscrimination gradients, with $\mathrm{S} 1$ versus $\mathrm{S} 2$ training (upper panel), the pigeons were predicted to respond relationally to S1 versus S5, to re- 
spond in accord with the absolute properties of the stimuli to S2 versus S3 and to S2 versus S6, and to respond at or slightly below chance levels to the remaining pairs. As was predicted, both birds responded relationally to S1 versus S5; however, both birds responded relationally to $\mathrm{S} 2$ versus S3 and to S2 versus S6. The Larger+ bird responded absolutely only to S0 versus $\mathrm{S} 7$ and to $\mathrm{S} 0$ versus S4, whereas the Smaller+ bird showed no significant absolute responding.

With S5 versus S6 training (lower panel), the postdiscrimination gradients predicted robust relational responding by both birds only to Pair S0 versus S7 and to Pair S3 versus S7; the prediction held true for the Smaller+ bird to S0 versus S7, but both birds responded absolutely to $\mathrm{S} 3$ versus $\mathrm{S} 7$. The postdiscrimination gradients also predicted robust absolute responding to Pair $\mathrm{S} 0$ versus S4 and to Pair S3 versus S4, but neither bird did so. For the remaining five testing pairs, gradient theory predicted absolute choice where relational choice resulted (S4 vs. S5 for the Smaller+ bird), no preference where absolute choice emerged (S2 vs. S3 for the Smaller+ bird), or no preference where relative choice emerged (S1 vs. S2 and S2 vs. S6 for the Smaller+ bird; S1 vs. S5 for the Larger+ bird).

In previous studies, transposition has often been evaluated with so-called far and near testing pairs. Transposition has often been found to decrease from near to far tests, a decrease that has been explained by a fall in the level of generalized associative strength as the testing stimuli become farther removed from the training stim- uli (reviewed by Reese, 1968, and Riley, 1968). Interestingly, our data also conformed with this pattern. For example, after $\mathrm{S} 1$ versus $\mathrm{S} 2$ training, relational responding was higher to Pair S2 versus S3 than to Pair S3 versus S4 for both birds; likewise, after S5 versus S6 training, both birds exhibited higher relational responding to Pair S4 versus S5 than to Pair S3 versus S4. Had we used only those testing pairs, we might have concluded that our one-pair training data corresponded to the predictions of Spence's (1937) theory. However, the other testing pairs that we included revealed an overall performance pattern that was quite different from that predicted by Spence's theory.

In short, the predictions of Spence's (1937) gradient theory fared rather poorly even after one-pair discrimination training. In several cases, relational responding emerged when it was not expected to do so.

\section{Two-Pair Training and Testing}

Two-pair discrimination training took a mean of 34.8 sessions, with a minimum of 7 sessions and a maximum of 72 sessions. Figure 12 shows the mean percentages of relational choices to all of the training and testing pairs in Experiment 1, as well as after both one- and two-pair training in Experiment 4.

Two-tailed binomial tests showed that mean relational responding to all of the training and testing pairs was significantly above chance level $(z \geq 2.7, p<.05)$. As is shown in Figure 12, relational responding to all of the testing pairs, except S3 versus S4, was higher after two-

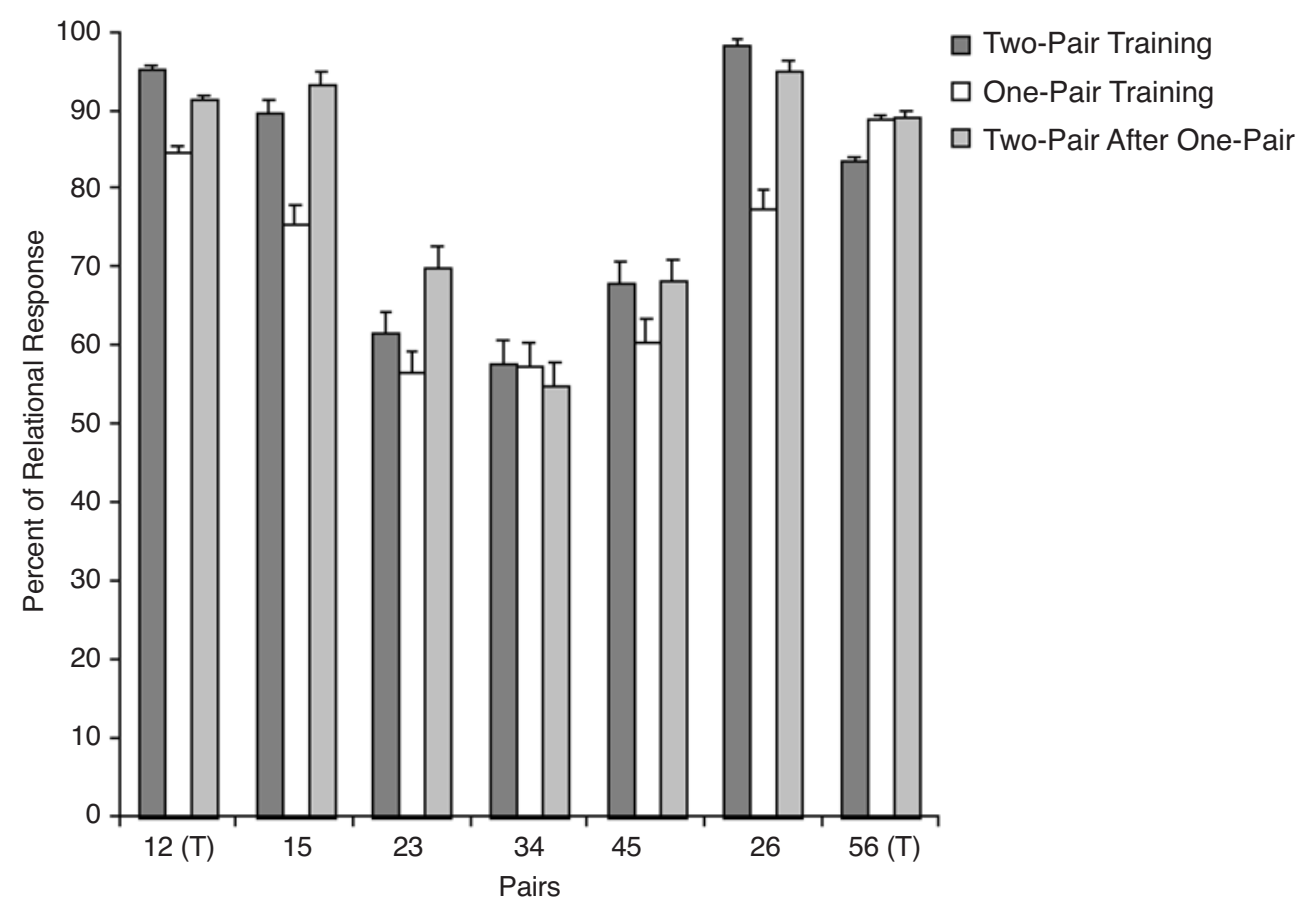

Figure 12. Mean percentages of relational responding after two-pair training alone (Experiment 1), after one-pair training alone, and after two-pair training following one-pair training (Experiment 4). The percentage of relational responding was significantly different from chance in all cases. 
pair training than after one-pair training. As well, relational responding was quite similar when two-pair training alone was given or when two-pair training followed one-pair training.

A repeated measures ANOVA of the Experiment 4 data, with training type (one-pair training or two-pair training) and testing pair as factors, showed a significant main effect of pair $[F(4,12)=9.60, p<.05]$. As in all of the earlier experiments, testing pairs could be ordered: $(\mathrm{S} 3$ vs. S4) $<($ S4 vs. S5 $)=($ S2 vs. S3 $)<($ S1 vs. S5 $)=$ (S2 vs. S6). More important, the ANOVA revealed a significant effect of Training Type $[F(1,3)=17.71, p<$ .05]; Tukey tests showed that responding to all of the testing pairs, except S3 versus S4 and S4 versus S5, was significantly higher after two-pair training than after one-pair training.

A between-subjects ANOVA of the Experiment 1 and Experiment 4 data, including training type (two-pair training or two-pair training after one-pair training) and testing pair as factors, also showed a significant effect of pair $[F(4,28)=122.45, p<.0001]$. But the ANOVA showed no significant effect of training type $[F(1,7)=$ $0.78, p=.38]$. The follow-up Tukey test revealed no significant differences between the experiments in relational responding to the different pairs.

We thus found that pigeons trained with only one pair of discriminative stimuli exhibited only modest relational responding. But if those same pigeons were later trained with two pairs of discriminative stimuli, their relational responding increased reliably and became comparable to the performance of the birds originally trained with two pairs of discriminative stimuli (Experiment 1). ${ }^{5}$

\section{GENERAL DISCUSSION}

\section{Empirical and Theoretical Summary}

In the present quartet of experiments, we found that pigeons' relational responding rose from one- to two- to four-pair discrimination training; the birds responded relationally to some of the testing pairs after one-pair training and to all of the testing pairs after two- and four-pair training. The reliable relational responding that we obtained after all three regimes cannot be predicted from the a priori excitatory and inhibitory stimulus generalization gradients that we chose for our simulations of Spence's (1937) theory of discrimination learning.

We chose those particular theoretical excitatory and inhibitory gradients so that they would closely accord with the empirical functions that have been reported in past studies of interdimensional and intradimensional stimulus generalization. Our theoretical gradients not only closely conformed to prior empirical gradients of excitation and inhibition, but also nicely fit our own training data depicted in Figures 6, 9, 10, and 11. Nevertheless, these same stimulus generalization gradients did not accord at all well with our pigeons' relational responding to the testing pairs. In each of the four experiments, clear failures of the model arose; the most glaring fail- ures involved our pigeons' responding relationally when Spence's (1937) theory expected the birds to respond in accord with the absolute associative histories of the discriminative stimuli.

As well, post hoc simulations using Shepard's (1987) stimulus generalization model failed to produce plausible excitatory and inhibitory gradients that could simultaneously account for our pigeons' relational responding to the testing pairs and their highly discriminative responding to the training pairs (Figure 7; additional simulations can be gotten from the corresponding author).

These collective failures strongly suggest that any theory of discrimination learning that is based solely on the associative histories of the individual discriminative stimuli cannot predict the relational responding that we observed in our experiments.

\section{Can Relational Theories Explain Our Data?}

It is one thing to conclude that Spence's (1937) theory of discrimination learning cannot explain our pigeons' testing behavior after single- and multiple-pair discrimination training, but it is quite another to conclude that our birds' relational responding must, therefore, be explained by a relational theory of discrimination learning. Just what candidate theories of relational choice are left to us if we reject Spence's absolute learning account?

Köhler (1918/1938) summarized the essential points of his relational account of transposition along the color dimension in the following manner:

(a) The individual colours appearing in a pair attain an inner union. ... [T] heir role in this union depends ... upon their places in the system they compose. (b) If their places with respect to each other are held constant but a variation is made in their absolute quality, . . . the perceived relationship will be transposed. (p. 221)

In other words, a relation between stimuli must exist in order for transposition to occur, but it need not be directly perceived; rather, a configuration of both discriminative stimuli is perceived holistically, and so long as this whole remains unchanged, the absolute values of the discriminative stimuli are irrelevant (also see Gulliksen \& Wolfle, 1938; Klüver, 1933; Köhler, 1929; Stevenson \& Bitterman, 1955; Washburn, 1926).

This relational notion was expressed more exactly by Riley (1958). He proposed that the effective discriminative stimulus in a two-choice task could be redefined as either (1) the ratio of one stimulus value to the other or (2) the ratio of the summed stimulus values to the background. Riley further proposed that a drop in transposition performance would occur if the stimulus ratios changed from training to testing - a form of stimulus generalization decrement.

Table 3 shows both of Riley's (1958) stimulus ratios for two-pair discrimination training along the size dimension. If the effective discriminative stimulus is redefined as the ratio of the smaller stimulus area to the larger stimulus area (upper row), Riley's (1958) ratio theory predicts weaker relational responding to Testing 
Table 3

Training (Italicized) and Testing Pairs in Experiment 2 Redefined as the Ratio of the Area of the Smaller Circle to the Area of the Larger Circle in a Pair (Ratio 1) or as the Ratio of the Area of the Background to the Sum of the Areas of the Two Circles (Ratio 2)

\begin{tabular}{cccccccc}
\hline Ratio & S1 vs. S2 & S1 vs. S5 & S2 vs. S3 & S3 vs. S4 & S4 vs. S5 & S2 vs. S6 & S5 vs. S6 \\
\hline 1 & 0.66 & 0.33 & 0.75 & 0.80 & 0.83 & 0.43 & 0.86 \\
2 & 36.84 & 10.08 & 18.56 & 10.88 & 6.36 & 7.38 & 4.06 \\
\hline
\end{tabular}

Pairs S1 versus S5 and S2 versus S6 than to the other three testing pairs, because these two ratios are the most different from the ratios of the training pairs; the ratios of Testing Pairs S1 versus S5 and S2 versus S6 fall outside the range of the two training pairs, whereas the ratios of the other three pairs fall inside the range of the two training pairs. Yet our experimental data clearly showed that pigeons exhibited even higher levels of relational responding to Testing Pairs S1 versus S5 and S2 versus $\mathrm{S} 6$ than to the other three testing pairs (Figures 5, 8 , and 10). Therefore, Ratio 1 must be an inappropriate predictor of relational responding.

If the effective discriminative stimulus is alternately defined as the ratio of the sum of the areas of both circles to the area of the background (lower row), Riley's (1958) ratio theory predicts similar levels of relational responding to Testing Pairs S1 versus S5 and S3 versus $\mathrm{S} 4$, because of their similar ratios. However, our data again do not accord with this prediction. Therefore, Ratio 2 must also be an inappropriate predictor, thereby questioning the utility of either variant of Riley's (1958) ratio theory for our data.

Another relational account, adaptation level theory (Capehart, Tempone, \& Hebert, 1969; James, 1953), hypothesizes that discrimination training leads to the establishment of an adaptation level, which represents the cumulative effect of all of the stimuli that have been given to the organism. Conventionally, the adaptation level is set at the geometric mean of the training values. During training, the organism is said to learn to approach the stimuli on one side of the adaptation level and to avoid the stimuli on the other side of the adaptation level. If, on the first testing trial, the values of both testing stimuli fall on each side of the training adaptation level, relational transfer is predicted. But if both values fall on the same side of the training adaptation level, random responding is predicted. (On later testing trials, after the organism has been familiarized with the testing stimuli, the adaptation level might shift, and relational responding might be predicted.)

Whatever the merits of adaptation level theory might be for predicting testing performance after one-pair discrimination training, in the case of multiple-pair discrimination training, adaptation level theory faces an extreme challenge. For example, it is impossible to establish a single adaptation level that can produce relational responding to both Training Pairs S1 + S2 - and S5 + S6at the same time. With this two-pair discrimination procedure, a single adaptation level must fall between S2 and S5; therefore, S1 and S2 each fall on the low side of the adaptation level, and S5 and S6 each fall on the high side of the adaptation level. If we assume that, after $\mathrm{S} 1+$ $\mathrm{S} 2$ - and $\mathrm{S} 5+\mathrm{S} 6$ - training, two adaptation levels must be established (one between S1 and S2 and the other between S5 and S6), then we need to specify how these two levels interact with one another to determine the outcome of a testing trial, such as S0 versus S7 (where S0 falls below and $\mathrm{S} 7$ falls above both adaptation levels) or $\mathrm{S} 3$ versus $\mathrm{S} 4$ (where $\mathrm{S} 3$ and $\mathrm{S} 4$ each fall above and below the two different adaptation levels). This problem requires an elaborate extension or revision of the theory, which, to our knowledge, has not been undertaken.

\section{Comparing Relational Responding After \\ One-, Two-, and Four-Pair Training}

Spence's (1937) theory of discrimination learning postulates that the algebraic sum of the excitatory and the inhibitory stimulus generalization gradients that develop around the training stimuli determines the choice of one testing stimulus over the other. As Shepard (1987) has shown, this idea essentially means that the similarity of a given testing stimulus to the previously reinforced and nonreinforced training stimuli can predict the choice of that stimulus in the testing situation. According to our data and simulations, the similarity of the training stimuli to the testing stimuli did not seem to play a prime role in the pigeons' relational responding; in all four of our experiments, the obtained profile of relational responding did not agree with that predicted by the excitatory and inhibitory stimulus generalization gradients (Figures $6,9,10$, and 11).

In a like manner, Riley's (1958) ratio theory postulates that relational responding is determined by the similarity of the pattern of testing stimuli as a whole to the pattern of training stimuli; the more dissimilar these two patterns are, the less relational responding should occur (Riley, 1958). But, those predictions, too, did not correspond with our data (see above for details).

Still, at least one form of stimulus similarity did reliably affect relational responding. In all of our experiments, the pigeons exhibited lower levels of relational responding to Testing Pairs S2 versus S3, S3 versus S4, and S4 versus S5 than to Testing Pairs S1 versus S5 and $\mathrm{S} 2$ versus S6 (see Figures 6, 9, and 11). The first three testing pairs comprise stimuli that are separated by one step along the logarithmic size scale, whereas the two final testing pairs comprise stimuli that are separated by four steps; in other words, the testing stimuli in the first 
three pairs were quite similar to each other, whereas the testing stimuli in the second two pairs were quite different from one another. Perhaps relational responding is affected primarily by the similarity of the two testing stimuli to one another: The more similar the testing stimuli, the more difficult it should be to determine which is the smaller (or larger), thus leading to poorer relational responding.

To test this additional relational idea, we conducted a series of regression analyses. In those analyses, we used the scaling function

$$
d=\log _{2}(y)-\log _{2}(x),
$$

where $d$ is the difference in scaled stimulus diameters, $x$ is the diameter of the smaller testing circle, and $y$ is the diameter of the larger testing circle. (This scaling function takes into account the fact that constant differences in circle diameter are not equivalently discriminable by using the logarithmic, rather than the absolute, values of circle diameter.)

When $d=0$ (i.e., when two stimuli are identical), choice responding is expected to be random; with larger values of $d$, the strength of relational responding is expected to increase. Therefore, we conceptualized the relation between predictor variable $d$ and the strength of relational responding to be a sigmoidal function; this function approximates a straight line when relational responding is weak, and it becomes increasingly sigmoidal when relational responding gets stronger (see Appendix B for details). The free-fitting parameter of this function, the gradient, represents the strength of relational responding: High values of the gradient correspond to strong relational responding, and low values correspond to weak relational responding.

We fit the data of each bird in each training regime, using nonlinear regression. To widen the interval of the tested values of the predictor, we included all of the pairs that were given to the different birds across the different training regimes. Therefore, there were 5 data points for each of the 4 birds given two-pair training (Experiment 1), 5 data points for 2 of the birds given four-pair training (Experiment 2), and 13 data points for the other 2 birds (Experiments 2 and 3); finally, there were 9 data points for each of the 4 birds given one-pair training (Experiment 4). Table 4 summarizes the gradient values for the best-fitting sigmoid functions of each bird's data, plus their goodness of fit as indicated by the root-mean square errors $\left(R M S_{\mathrm{e}} \mathrm{s}\right)$.

Fitting the data for the pigeons that underwent onepair training, we obtained quite low gradient parameters, suggesting weak and gradual relational responding. In addition, quite high $R M S_{\mathrm{e}}$ values suggested that the difference between the sizes of the testing stimuli, $d$, was a weak predictor of testing performance in this group.

In the two-pair training group, the values of the gradient parameter were notably higher than those in the onepair training group, suggesting that relational responding in the two-pair group was stronger. For 2 of the 4
Table 4

Fits of the Proportions of Relational Responding After OnePair, Two-Pair, and Four-Pair Discrimination Training

\begin{tabular}{lcc}
\hline & \multicolumn{2}{c}{ Parameters } \\
\cline { 2 - 3 } Training Type & Gradient & $R M S_{\mathrm{e}}$ \\
\hline One-Pair Training (Experiment & 4 ; 9 Data Points) \\
S1 + S2 - training & 0.269 & 0.144 \\
S1 - S2 + training & 0.134 & 0.214 \\
S5+ S6 - training & 0.024 & 0.138 \\
S5 - S6 + training & 0.256 & 0.287 \\
Two-Pair Training (Experiment 1; 5 Data Points) \\
Smaller+, Bird 1 & 3.233 & 0.0312 \\
Smaller+, Bird 2 & 1.518 & 0.115 \\
Larger+, Bird 1 & 1.429 & 0.096 \\
Larger+, Bird 2 & 1.429 & 0.119 \\
\multicolumn{3}{c}{ Four-Pair Training (Experiments 2 and 3) } \\
Smaller+, Bird 1 (13 data points) & 4.533 & 0.0312 \\
Smaller+, Bird 2 (5 data points) & 3.403 & 0.049 \\
Larger+, Bird 1 (13 data points) & 3.066 & 0.071 \\
Larger+, Bird 2 (5 data points) & 2.109 & 0.056 \\
\hline
\end{tabular}

Note-All of the testing pairs were included in the fits; therefore, the number of data points differed across the three different training regimes.

birds (Bird 1 Smaller+ and Bird 1 Larger+), our function produced good fits, as indicated by the low $R M S_{\mathrm{e}}$ values; for the other 2 birds (Bird 2 Smaller + and Bird 2 Larger+), however, the fits were poorer. Thus, $d$ became a better, albeit not strong, predictor of testing performance after two-pair training, as compared with one-pair training.

Finally, for the 4 birds that were exposed to four-pair training, the values of the gradient parameter were quite high, suggesting that these birds exhibited very strong relational responding. In addition, the $R M S_{\mathrm{e}}$ values indicated an excellent fit for all 4 birds, confirming that the difference in the sizes of the testing stimuli excellently predicted relational responding to the novel testing pairs after four-pair training.

Our simulations disclosed that both the strength of relational responding and the reliability of our predictor rose with increases in the number of training pairs. Thus, our predictor captures the rise in strength of relational responding from one-pair to two-pair to four-pair training. Presumably, as more training pairs are presented, absolute cues (such as the similarity of the training stimuli to the testing stimuli) become less salient, and relational cues (such as the similarity of the testing stimuli to each other) become more salient. We hope that the present data will encourage the investigation of relational responding in respect to the number of training pairs.

We do wish to note some limitations in the present data. First, the increasing strength of relational responding across the three training regimes may to some extent, be captured by Spence's (1937) theory. Figure 13 shows the mean level of relational responding across the five common testing pairs ( 11 vs. S5, S2 vs. S3, S3 vs. S4, S4 vs. $\mathrm{S} 5$, and S2 vs. S6) in Test 3 and the mean level of rela- 


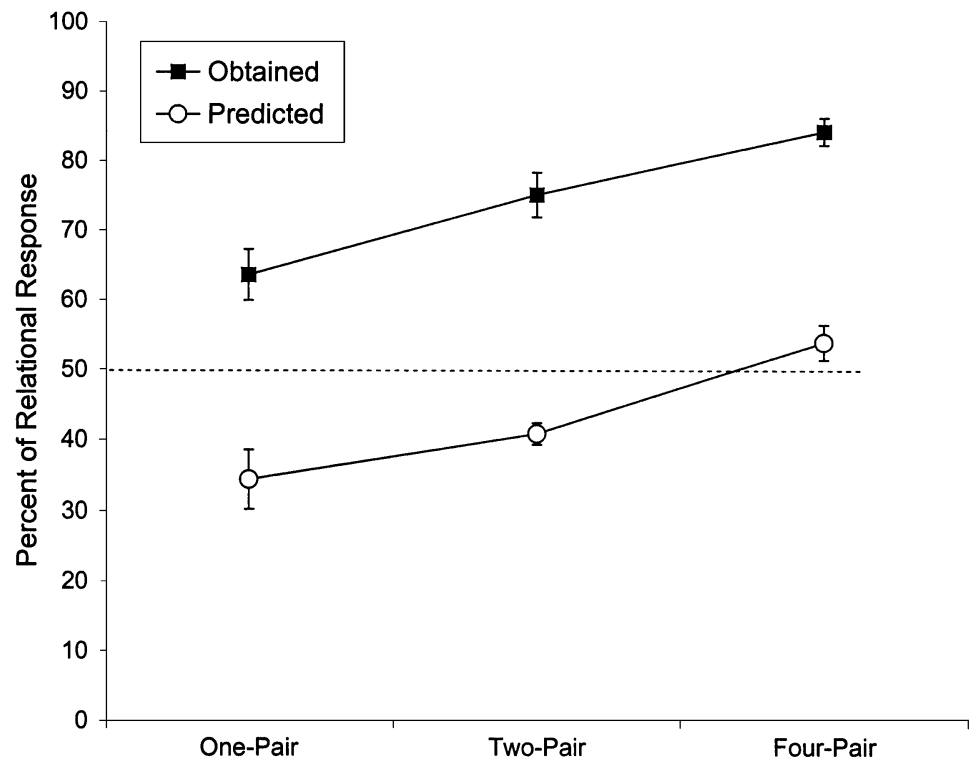

Figure 13. Mean percentages of relational responding across the five common testing pairs in Test 3 after one-, two-, and four-pair discrimination training in Experiments 1, 2, and 4 (black squares) or as predicted by the postdiscrimination gradients (white circles).

tional responding predicted by Spence's postdiscrimination gradients. The pigeons' mean level of relational responding increased from $64 \%$ in one-pair training to $75 \%$ in two-pair training to $84 \%$ in four-pair training. The mean levels of relational responding that were predicted by Spence's model increased as well: With more excitatory and inhibitory stimulus generalization gradients interacting along the size dimension, there was a higher probability of predicting relational responding for any given testing pair (see Figures 8 and 9). However, there was a large and constant (on average, $31 \%$ ) disparity between predicted and obtained relational responding: Indeed, in the case of four-pair training, the predicted level of relational responding was still at chance (54\%). In other words, although Spence's absolute theory correctly predicted an increase in relational responding with increases in the number of training pairs, the predicted levels of relational responding fell far below the empirical data for all three of the training regimes. ${ }^{6}$

The second limitation is related to the range of the scaled size differences. The scaled size differences in the five testing pairs that were common to all of the training conditions fell between 0.0 and 0.5 (S4 vs. S5, S3 vs. S4, and $\mathrm{S} 2$ vs. S3) and between 1.0 and 2.0 (S1 vs. S5 and S2 vs. S6). The fit that we presented above (Table 4) incorporated all of the testing pairs, some of which were not presented across all of the training conditions (e.g., S0 vs. S7, a scaled size difference of 3.0). However, we also found similar results when we fit the pigeons' testing performance, using only the five data points that were common to all three training regimes. (These fits can be obtained from the corresponding author.)
Finally, it is possible that some other scaling functions might produce similar or even better fits of our data. Unfortunately, the limited range of the testing pairs does not permit us to evaluate these alternatives at the present time. Further experiments in which larger sets of testing pairs are used should help clarify this issue. Nevertheless, our data do allow us to conclude that (1) the difference in size between the testing stimuli exerts strong control over pigeons' relational test responding and (2) this relational stimulus control is stronger when training involves multiple pairs of training stimuli.

\section{Conclusions}

Studies of transposition and related discriminationlearning phenomena have often yielded data that do not unequivocally support either Spence's (1937) theory or relational discrimination accounts. In some experiments, the relational responding obtained was believed to be impossible to explain by Spence's absolute theory (Johnson \& Zara, 1960; Lawrence \& DeRivera, 1954; Marsh, 1967), but in other experiments, the behavioral evidence seemed to provide strong support for Spence's theory (Ehrenfreund, 1952; Guttman \& Kalish, 1956; Hanson, 1959; Hearst, 1969; Honig, 1962; Honig \& Urcuioli, 1981; Purtle, 1973). Despite this mixed empirical record, Spence's theory has endured as the most prominent account of transposition and relational stimulus control.

Our own data provide strong evidence against Spence's (1937) theory; indeed, even in the case of one-pair discrimination training, the pattern of relational responding that we obtained could not be predicted by interacting stimulus generalization gradients of excitation and inhi- 
bition (Figure 11). Note that we are not playing favorites; our data do not accord with existing relational accounts either. Relational theories predict that, if the testing configuration differs from the training configuration, the organism should respond at chance levels, or even in accord with the absolute properties of the stimuli. Yet we found that many of the testing pairs that differed dramatically from the training pairs actually supported the highest levels of relational responding.

Contrary to all of the available absolute and relational theories, we found that the similarity of the two testing stimuli to one another strongly affected relational responding: the more dissimilar the two stimuli, the higher the relational responding (Table 4). To the best of our knowledge, this is the first evidence that the similarity of the testing stimuli can control relational responding; indeed, the similarity of the testing stimuli to the training stimuli may be largely irrelevant, especially in case of multiple-pair discrimination training. It is certainly possible that absolute cues interact with relational cues when only one training pair is presented. However, given the high proportion of variance that was accounted for by the similarity of the testing stimuli after two- and, especially, four-pair discrimination training, there is very little room for the effective operation of absolute associative tendencies in the case of multiple-pair discrimination training (Table 4).

Transposition has conventionally been assessed after one-pair training with testing stimuli that are progressively distant from the training pair and separated by one dimensional step: from near to far tests of transposition. The common finding in such tests is a decrease in relational responding from the near to the far test. Our own one-pair testing data replicated this finding (cf. Figure 10). But when we included testing pairs that were separated by more than one step, a dramatically different pattern emerged: Even in the case of one-pair training, the birds exhibited relational responding, contrary to the predictions of the postdiscrimination gradients (cf. Figure 10). Thus, pigeons exhibit relational stimulus control in the same basic task for which Spence's (1937) theory of absolute responding was originally devised. We believe that our data thus represent strong support for the idea that animals are indeed capable of relational responding (cf. Mackintosh, 2000; Wills \& Mackintosh, 1999) and reveal two important variables that strongly affect relational responding in pigeons: the number of training pairs that are given and the similarity of the testing stimuli to one another. We hope that our data and analyses will rekindle fresh empirical and theoretical investigations into an area that has unfortunately been neglected by psychological scientists for several decades.

\section{REFERENCES}

Blough, D. (1969). Generalization gradient shape and summation in steady-state tests. Journal of the Experimental Analysis of Behavior, 12, 91-104.

BLOUGH, D. (1975). Steady-state data and a quantitative model of op- erant generalization and discrimination. Journal of Experimental Psychology: Animal Behavior Processes, 104, 3-21.

Capehart, J., Tempone, V. J., \& Hebert, J. (1969). A theory of stimulus equivalence. Psychological Review, 76, 405-418.

Cheng, K. (1999). Spatial generalization in honeybees confirms Shepard's law. Behavioural Processes, 44, 309-316.

Cheng, K., \& Spetch, M. L. (2002). Spatial generalization and peak shift in humans. Learning \& Motivation, 33, 358-389.

Cheng, K., Spetch, M. L., \& Johnston, M. (1997). Spatial peak shift and generalization in pigeons. Journal of Experimental Psychology: Animal Behavior Processes, 23, 469-481.

CoOK, R. G. (2002). The structure of pigeon multiple-class same-different learning. Journal of the Experimental Analysis of Behavior, 78, $345-$ 364.

Cook, R. G., Cavoto, K. K., \& Cavoto, B. R. (1995). Same/different texture discrimination and concept learning in pigeons. Journal of Experimental Psychology: Animal Behavior Processes, 21, 253-260.

Cook, R. G., Katz, J. S., \& CAvoto, B. R. (1997). Pigeon same-different concept learning with multiple stimulus classes. Journal of Experimental Psychology: Animal Behavior Processes, 23, 417-433.

EHRENFREUND, D. (1952). A study of the transposition gradient. Journal of Experimental Psychology, 43, 81-87.

FaGot, J., Wasserman, E. A., \& Young, M. E. (2001). Discriminating the relation between relations: The role of entropy in abstract conceptualization by baboons and humans. Journal of Experimental Psychology: Animal Behavior Processes, 27, 316-328.

Gonzalez, R. C., Gentry, G. V., \& BitTerman, M. E. (1954). Relational discrimination of intermediate size in the chimpanzee. Journal of Comparative \& Physiological Psychology, 47, 385-388.

Gulliksen, H., \& Wolfle, D. L. (1938). A theory of learning and transfer: I. Psychometrika, 3, 127-149.

Guttman, N., \& Kalish, H. I. (1956). Discriminability and stimulus generalization. Scientific American, 51, 77-82.

HANSON, H. M. (1959). Effects of discrimination training on stimulus generalization. Journal of Experimental Psychology, 58, 321-334.

HEARST, E. (1969). Excitation, inhibition, and discrimination learning. In N. J. Mackintosh \& W. K. Honig (Eds.), Fundamental issues in associative learning: Proceedings of a symposium held at Dalhousie University, Halifax, June 1968 (pp. 1-41). Halifax: Dalhousie University Press.

Hebert, J. A., \& Krantz, D. L. (1965). Transposition: A reevaluation. Psychological Bulletin, 63, 244-257.

HeinemanN, E. G., \& RuDOLPH, R. L. (1963). The effect of discriminative training on the gradient of stimulus-generalization. American Journal of Psychology, 76, 653-658.

HERRNSTEIN, R. J. (1990). Levels of stimulus control: A functional approach. Cognition, 37, 133-166.

Honig, W. K. (1962). Prediction of preference, transposition, and transposition-reversal from the generalization gradient. Journal of Experimental Psychology, 64, 239-248.

Honig, W. K., Boneau, C. A., Burstein, K. R., \& Pennypacker, H. S. (1963). Positive and negative generalization gradients obtained after equivalent training conditions. Journal of Comparative \& Physiological Psychology, 56, 111-116.

Honig, W. K., \& Urcuioli, P. J. (1981). The legacy of Guttman and Kalish (1956): 25 years of research on stimulus generalization. Journal of the Experimental Analysis of Behavior, 36, 405-445.

JAMES, W. (1953). An application of Helson's theory of adaptation level to the problem of transposition. Psychological Review, 60, 345-351.

JENKINS, H. M. (1965). Generalization gradients and the concept of inhibition. In D. I. Mostofsky (Ed.), Stimulus generalization (pp. 5561). Stanford, CA: Stanford University Press.

Johnson, R. C., \& ZARA, R. C. (1960). Relational learning in young children. Journal of Comparative \& Physiological Psychology, 53, 594-597.

KATZ, J. S., Wright, A. A., \& Bachevalier, J. (2002). Mechanisms of same/different abstract-concept learning by rhesus monkeys (Macaca mulatta). Journal of Experimental Psychology: Animal Behavior Processes, 28, 358-368.

KLÜVER, H. (1933). Behavior mechanisms in monkeys. Chicago: University of Chicago Press. 
KöHLER, W. (1929). Gestalt psychology. New York: Liveright.

KöHLER, W. (1938). Simple structural functions in the chimpanzee and in the chicken. In W. D. Ellis (Ed. \& Trans.), A source book of Gestalt psychology (pp. 217-227). London: Routledge \& Kegan Paul. (Original work published 1918)

Larsen, A., \& Bundesen, C. (1978). Size scaling in visual pattern recognition. Journal of Experimental Psychology: Human Perception \& Performance, 4, 1-20.

LaWrence, D. H., \& DeRivera, J. (1954). Evidence for relational transposition. Journal of Comparative \& Physiological Psychology, 47, 465-471.

LUCE, R. D. (1959). Individual choice behavior: A theoretical analysis. New York: Wiley.

Mackintosh, N. J. (1974). The psychology of animal learning. London: Academic Press.

Mackintosh, N. J. (2000). Abstraction and discrimination. In C. Heyes $\&$ L. Huber (Eds.), The evolution of cognition (pp. 123-142). Cambridge, MA: MIT Press.

Mark, R. F., \& MaXwell, A. (1969). Circle size discrimination and transposition behaviour in cichlid fish. Animal Behaviour, 17, 155158.

MARSH, G. (1967). Relational learning in the pigeon. Journal of Comparative \& Physiological Psychology, 64, 519-521.

MARSH, G. (1972). Prediction of the peak shift in pigeons from gradients of excitation and inhibition. Journal of Comparative \& Physiological Psychology, 81, 262-266.

MorGan, C. L. (1894). An introduction to comparative psychology. London: Walter Scott.

Pasnak, R., \& KurTz, S. L. (1987). Brightness and size transposition by rhesus monkeys. Bulletin of the Psychonomic Society, 25, 109112.

PurtLe, R. B. (1973). Peak shift: A review. Psychological Bulletin, 80, 408-421.

ReEse, H. W. (1968). The perception of stimulus relation: Discrimination learning and transposition. New York: Academic Press.

Rescorla, R. A. (1969). Pavlovian conditioned inhibition. Psychological Bulletin, 72, 77-94.

RILEY, D. A. (1958). The nature of the effective stimulus in animal discrimination learning: Transposition reconsidered. Psychological Review, 65, 1-7.

RILEY, D. A. (1968). Discrimination learning. Boston: Allyn \& Bacon.

Riley, D. A., Goggin, J. P., \& Wright, D. C. (1963). Training level and cue separation as determiners of transposition and retention in rats. Journal of Comparative \& Physiological Psychology, 56, 1044-1049.

RiLEy, D. A., Ring, K., \& Thomas, J. (1960). The effect of stimulus comparison on discrimination learning and transposition. Journal of Comparative \& Physiological Psychology, 53, 415-421.

RILLING, M. (1977). Stimulus control and inhibitory processes. In W. K. Honig \& J. E. R. Staddon (Eds.), Handbook of operant behavior (pp. 432-480). Englewood Cliffs, NJ: Prentice-Hall.

RILLING, M., \& BUDNIK, J. E. (1975). Generalization of excitation and inhibition after different amounts of training of an avoidance baseline. Journal of the Experimental Analysis of Behavior, 23, 207-215.

Roberts, S., \& PASHLer, H. (2000). How persuasive is a good fit? A comment on theory testing. Psychological Review, 107, 358-367.

SHEPARD, R. N. (1987). Toward a universal law of generalization for psychological science. Science, 237, 1317-1323.

Sherman, M., \& StrunK, J. (1964). Transposition as a function of single versus double discrimination training. Journal of Comparative \& Physiological Psychology, 58, 449-450.

SIDMAN, M. (1961). Stimulus generalization in an avoidance situation. Journal of the Experimental Analysis of Behavior, 4, 157-169.

SPENCE, K. W. (1937). The differential response in animals to stimuli varying within a single dimension. Psychological Review, $\underline{\mathbf{4 4}, 430-}$ 444.

Stevenson, H. W., \& Bitterman, M. E. (1955). The distance-effect in the transposition of intermediate size by children. American Journal of Psychology, 68, 274-279.

THOMPSON, R. K. R., \& ODEN, D. L. (2000). Categorical perception and conceptual judgments by nonhuman primates: The paleological monkey and the analogical ape. Cognitive Science, 24, 363-396.

WaShburn, M. F. (1926). The animal mind. New York: Macmillan. Wasserman, E. A., Hugart, J. A., \& Kirkpatrick-Steger, K. (1995). Pigeons show same-different conceptualization after training with complex visual stimuli. Journal of Experimental Psychology: Animal Behavior Processes, 21, 248-252.

WILLS, S., \& MACKINTOSH, N. J. (1999). Relational learning in pigeons? Quarterly Journal of Experimental Psychology, 52B, 31-52.

WrIGHT, A. A. (1997). Concept learning and learning strategies. Psychological Science, 8, 119-123.

Wright, A. A., CoOK, R. G., Rivera, J. U., Sands, S. F., \& Delius, J. D. (1988). Concept learning by pigeons: Matching-to-sample with trial-unique video picture stimuli. Animal Learning \& Behavior, 16, 436-444.

Young, M. E., \& Wasserman, E. A. (2001). Evidence for a conceptual account of same-different discrimination learning in the pigeon. Psychonomic Bulletin \& Review, 8, 677-684.

\section{NOTES}

1. According to Blough's (1975) generalization theory, training stimuli accumulate associative strength in accord with the Rescorla-Wagner equations; this strength is then transferred along the dimension in the form of stimulus generalization decrement. However, Blough's model was developed for the special case in which $\mathrm{S}$ - was occasionally reinforced; therefore, Shepard's (1987) theory was more appropriate for our purposes.

2 . We again considered the same variations in the shapes of the generalization gradients, as shown in Figures 3 and 4, and once more found little difference in the resulting predictions. Hereafter, we will include only the case in which the excitatory gradient is taller and narrower than the inhibitory gradient. Simulations using Shepard's (1987) model again produced unrealistic gradients, as in Experiment 1. Hereafter, no additional post hoc simulations will be presented; they can be obtained from the corresponding author.

3. Caution should be exerted in interpreting this analysis, given the possible known issues with cross-experiment comparisons. Indeed, two-pair training was, on average, briefer than four-pair training (26.3 and 33.8 sessions, respectively), and it resulted in slightly lower overall training performance during testing $(89.2 \%$ and $91.7 \%$, respectively). Although overtraining may increase the level of relational responding (Riley, Goggin, \& Wright, 1963), it might be noted that, in our case, the pigeons in both groups were trained to criterion and were not overtrained. In addition, at least some experimental evidence suggests that fast-learning subjects actually show stronger transposition than slowlearning subjects (reviewed by Reese, 1968, pp. 140-146).

4. In all of the cases illustrated in Figure 2, the postdiscrimination gradients predicted higher responding to the previously reinforced stimulus than to the adjacent novel stimulus: with $\mathrm{S} 0$ versus $\mathrm{S} 1$ after $1+2-$ training, with $\mathrm{S} 2$ versus $\mathrm{S} 3$ after $1-2+$ training, with $\mathrm{S} 4$ versus $\mathrm{S} 5$ after $\mathrm{S} 5+\mathrm{S} 6-$ training, and with $\mathrm{S} 6$ versus $\mathrm{S} 7$ after $\mathrm{S} 5-\mathrm{S} 6+$ training. No positive peak shift was predicted because of the large distances between the stimuli on the logarithmic size scale and because of the narrow gradients that were necessary to support effective discrimination learning between Training Pair S1 and S2 and between Training Pair S5 and S6. Had the testing circles been more similar to one another, the postdiscrimination gradients would have predicted a positive peak shift. In fact, the absence of a positive peak shift to the testing stimuli in the predicted gradients does not constitute a serious limitation, because positive peak shift is not an inevitable consequence of intradimensional discrimination training (Hearst, 1969; Honig, 1962; Mackintosh, 1974; Purtle, 1973; Rilling, 1977).

5. Again, caution should be taken in interpreting these data. In some experiments, transposition has been found to increase with repeated testing. It should be pointed out, however, that the experimental evidence is not consistent. As well, no increase or decrease in transposition over the course of a testing session has yet been reported (reviewed by Reese, 1968, pp. 24-31). 
6. It is possible to design an experiment in which Spence's (1937) theory predicts a decrease in relational responding with an increase in the number of training pairs. Suppose that one-pair training involves $\mathrm{S} 1+$ $\mathrm{S} 2-$ training, two-pair training involves $\mathrm{S} 1+\mathrm{S} 2-$ and $\mathrm{S} 2+\mathrm{S} 3-$ training, and four-pair training involves $\mathrm{S} 1+\mathrm{S} 2-, \mathrm{S} 2+\mathrm{S} 3-, \mathrm{S} 3+\mathrm{S} 4-$, and $\mathrm{S} 4+\mathrm{S} 5-$ training. The critical testing pair is S5 versus $\mathrm{S} 6$. Because re- lationally correct Stimulus S5 gradually comes closer to the stimulus that is not reinforced during training, we expect relational responding to be lower after four-pair training than after one-pair training. Simulations using the same gradients as those shown in Figure 2 predict an average of relational responding in Pair S5 versus S6 to be $50 \%$ after one-pair training, $47 \%$ after two-pair training, and $36 \%$ after four-pair training.

\section{APPENDIX A \\ Shepard's Stimulus Generalization Function}

Shepard's (1987) stimulus generalization function for one-dimensional space can be written as

$$
G(d)=e^{-c * d},
$$

where $G$ is the generalization (or similarity judgment) function, $c$ is a steepness parameter, and $d$ is the distance between the training stimulus, $x$, and the testing stimulus, $y$, defined as

$$
d=|x-y|^{i},
$$

where $i$ is a free parameter.

According to Spence's (1937) theory, excitatory and inhibitory gradients have two important properties that can change the shape of the postdiscrimination gradient - namely, height and steepness. However, from Equations 1 and 2, it follows that $G(d)=1$ when $x=y$ (i.e., when we calculate the similarity of the stimulus to itself) and that $0<G(d)<1$ otherwise. So the height of any stimulus generalization gradient obtained with Shepard's (1987) generalization function will always be the same and equal to 1.00 . The steepness of the stimulus generalization gradient can vary; therefore, we concentrated on this parameter.

We assumed that all possible excitatory and inhibitory stimulus generalization gradients for a given kind of stimulation ought to be selected from the same family of functions, because excitation and inhibition are conventionally considered to be diametrically opposite behavioral processes (Rescorla, 1969; Spence, 1937). Thus, three free parameters were allowed: $i$ for the general shape of the stimulus generalization function, $c_{\mathrm{E}}$ for the steepness of the excitatory function, and $c_{\mathrm{I}}$ for the steepness of the inhibitory function.

We first simulated these excitatory and inhibitory stimulus generalization gradients for all of the training and testing scores. We then algebraically summed the two gradients to produce a single postdiscrimination gradient. Next, we predicted the average pigeon's discrimination performance to the training and testing pairs, using Luce's (1959) choice rule

$$
p=e^{A * G(x)} /\left[e^{A * G(x)}+e^{A * G(y)}\right],
$$

where $G(x)$ and $G(y)$ are the values of the generalization function for Stimuli $x$ and $y$, respectively, and $A$ is a bias parameter. This choice rule produces a higher probability of selecting the response associated with Stimulus $x$ as the strength of their response, $G(x)$, increases relative to the strength of the alternative response, $G(y)$. The exponentials produce a sigmoidal, rather than a linear, relationship between the strength of the response and the probability of selecting this response.

We selected the best-fitting result, using the least-square method. Finally, we decided whether or not to adjust the bias parameter of Luce's (1959) choice rule; if a change in this bias parameter produced a better fit to the experimental data, we saved those results. Note that any change in bias did not change the underlying stimulus generalization gradients; it only raised or lowered the predicted levels of choice responding. 


\section{APPENDIX B}

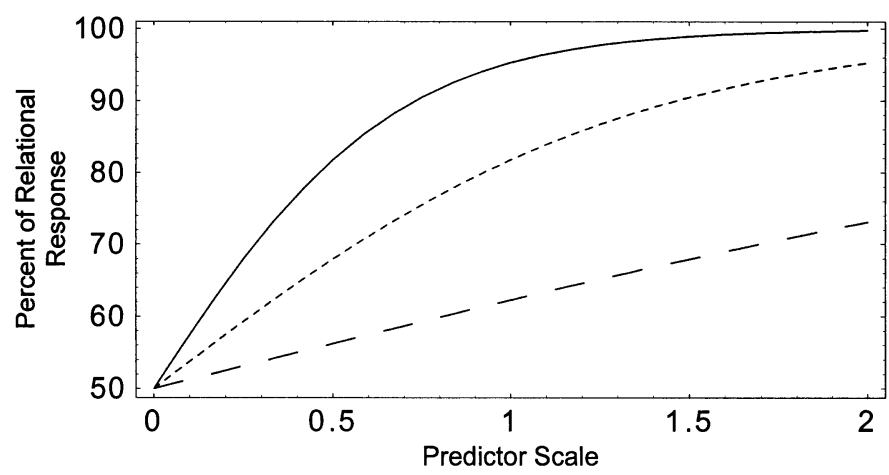

Figure B1. Sigmoid fits of hypothetical data. The dashed line represents the fit with a low gradient parameter, the dotted line represents the fit with an intermediate gradient parameter, and the smooth line represents the fit with a high gradient parameter $(0.5,1.5$, and 3 , respectively). Note that because the boundary parameter is set to zero, both curves intersect the $y$-axis at the point that corresponds to random responding $(50 \%)$. Thus, the graph illustrates only the half of a sigmoid curve that corresponds to positive values of the predictor, $d$.

To evaluate the connection between relational responding and the difference in size of the testing stimuli, we fit the data, using the following equation:

$$
\frac{1}{1+e^{- \text {gradient } \times(\text { predictor }+ \text { boundary })}},
$$

where gradient and boundary are free-fitting parameters and predictor is the value of $d$, the logarithmic difference in the two circle sizes (see Equation 2).

The values of the free-fitting parameters reflect the choice behavior of the subjects. The boundary parameter represents the point of maximal inflection for the sigmoid. In our case, it is theoretically constrained to zero (cf. Figure B1): When $d=0$, the two circles are indistinguishable, and chance responding is expected. In addition, exploratory fits showed that the best-fitting value of the boundary parameter did not differ significantly from zero. So, in the present series of fits, the boundary parameter was set to zero and was not allowed to vary. Thus, Equation B1 can be rewritten as

$$
\frac{1}{1+e^{- \text {gradient } \times \text { predictor }}} \text {. }
$$

The gradient parameter represents the strength of relational responding (Figure B1). When the gradient of the best-fitting sigmoid is high (smooth line), the subjects exhibit a strong tendency to respond in accord with the previously learned relation and a low tendency to respond in accord with the absolute properties of the stimuli. When the gradient is intermediate (dotted line) or low (dashed line), the subjects show a more gradual transition from chance to relational responding. 